OPEN ACCESS

Edited by:

Trevor Chung-Ching Chen, National Taiwan Normal University,

Taiwan

Reviewed by:

Tsang-Hai Huang,

National Cheng Kung University,

Taiwan

Sataro Goto,

Juntendo University, Japan

Yu-Hsiang Hsu,

National Cheng Kung University,

Taiwan

${ }^{*}$ Correspondence:

Lunhao Bai

cmublh@163.com

Specialty section:

This article was submitted to

Exercise Physiology,

a section of the journal

Frontiers in Physiology

Received: 25 March 2019

Accepted: 02 August 2019

Published: 20 August 2019

Citation:

Zhang $H$, Ji L, Yang Y, Wei Y,

Zhang $X$, Gang $Y, L U J$ and Bai $L$

(2019) The Therapeutic Effects

of Treadmill Exercise on Osteoarthritis

in Rats by Inhibiting

the HDAC3/NF-KappaB Pathway

in vivo and in vitro.

Front. Physiol. 10:1060.

doi: 10.3389/fphys.2019.01060

\section{The Therapeutic Effects of Treadmill Exercise on Osteoarthritis in Rats by Inhibiting the HDAC3/NF-KappaB Pathway in vivo and in vitro}

\author{
He Zhang ${ }^{1}$, Lu Ji ${ }^{2}$, Yue Yang ${ }^{1}$, Yingliang Wei ${ }^{1}$, Xiaoning Zhang ${ }^{3}$, Yi Gang ${ }^{4}$, Jinghan Lu ${ }^{1}$ \\ and Lunhao Bai ${ }^{*}$ \\ 1 Department of Orthopedic Surgery, Shengjing Hospital, China Medical University, Shenyang, China, ${ }^{2}$ Department \\ of Gynecology and Obstetrics, Shengjing Hospital, China Medical University, Shenyang, China, ${ }^{3}$ Department \\ of Anesthesiology Department, Shengjing Hospital, China Medical University, Shenyang, China, ${ }^{4}$ Department of Orthopedic \\ Surgery, Panjin Central Hospital, Panjin, China
}

Osteoarthritis $(\mathrm{OA})$ is a disease characterized by non-bacterial inflammation. Histone deacetylase 3 (HDAC3) is a crucial positive regulator in the inflammation that leads to the development of non-OA inflammatory disease. However, the precise involvement of HDAC3 in OA is still unknown, and the underlying mechanism of exercise therapy in OA requires more research. We investigated the involvement of HDAC3 in exercise therapytreated OA. Expression levels of HDAC3, a disintegrin and metalloproteinase with thrombospondin motifs-5 (ADAMTS-5), matrix metalloproteinase-13 (MMP-13), HDAC3 and nuclear factor-kappaB (NF-kappaB) were measured by western blotting, reverse transcription polymerase chain reaction (RT-PCR) and immunohistochemistry. Cartilage damage and OA evaluation were measured by hematoxylin and eosin staining and Toluidine blue $O$ staining according to the Mankin score and OARSI score, respectively. We found that moderate-intensity treadmill exercise could relieve OA. Meanwhile, the expression of HDAC3, MMP-13, ADAMTS-5 and NF-kappaB decreased, and collagen II increased in the OA + moderate-intensity treadmill exercise group (OAM) compared with the OA group (OAG) or OA + high- or low-intensity treadmill exercise groups (OAH or OAL). Furthermore, we found the selective HDAC3 inhibitor RGFP966 could also alleviate inflammation in $\mathrm{OA}$ rat model through inhibition of nuclear translocation of NF-kappaB. To further explore the relationship between HDAC3 and NF-kappaB, we investigated the change of NF-kappaB relocation in IL-1 $\beta$-treated chondrocytes under the stimulation of RGFP966. We found that RGFP966 could inhibit the expression of inflammatory markers of OA via regulation of HDAC3/NF-kappaB pathway. These investigations revealed that RGFP966 is therefore a promising new drug for treating OA.

Keywords: osteoarthritis (knee), HDAC3, inflammation, treadmill exercise, NF-kappaB, RGFP966

Abbreviations: ADAMTS-5, a disintegrin and metalloproteinase with thrombospondin motifs-5; CG, control group; DMEM, Dulbecco's modified Eagle medium; FBS, fetal bovine serum; GAG, glycosaminoglycan; HDACs, histone deacetylases; HDAC3, histone deacetylase 3; IHC, immunohistochemistry; IL-1 $\beta$, interleukin-1 $\beta$; MIA, monosodium iodoacetate; MMP-13, matrix metalloproteinase-13; NF-kappaB, nuclear factor-kappaB; OA, osteoarthritis; OAG, OA group; OAL, OA + low-intensity of treadmill exercise group; OAM, OA + moderate-intensity of treadmill exercise group; OAH, $\mathrm{OA}+$ high-intensity of treadmill exercise group; ROS, reactive oxygen species; TNF- $\alpha$, tumor necrosis factor- $\alpha$. 


\section{INTRODUCTION}

Osteoarthritis (OA) is a highly prevalent degenerative disease worldwide. In 2015, almost 54.4 million adults were diagnosed with OA in the United States (Cheng et al., 2010); this number is increasing rapidly. $\mathrm{OA}$ is a multi-factor disease, and obesity, age, gender, and abnormal loading of joints are all able to stimulate production of inflammatory mediators secreted by chondrocyte and synovium including IL-1 $\beta$, ADAMTS- 5 and MMP-13, which contribute to OA characterized by cartilage erosion, subchondral bone formation, and joint swelling (Ashkavand et al., 2013; Berenbaum, 2013; Mobasheri and Batt, 2016). Recently, exercise therapy has received considerable attention for treating OA, as an alternative to surgery (McAlindon et al., 2014). Gentle exercise can prevent subchondral cyst formation and osteoclast activity in OA development (Iijima et al., 2015); moderateintensity exercise can also promote glycosaminoglycan (GAG) content in the cartilage and prevent cartilage damage including extracellular matrix loss, chondrocytes apoptosis, inflammation development, and osteophyte formation (Cifuentes et al., 2010; $\mathrm{Ni}$ et al., 2013; Boudenot et al., 2014). Conversely, improper exercise can aggravate OA (Franciozi et al., 2013). However, the mechanism of different intensities of exercise involved in the variable effect on $\mathrm{OA}$ is still unknown.

Histone deacetylases are a family of proteins that regulate acetylation of proteins, and can be divided into four groups: class I HDACs, class II HDACs, Sirtuins and HDAC11 (De Ruijter et al., 2003). HDACs largely contribute to the pathological process of $\mathrm{OA}$; levels of $\mathrm{HDAC1}, 2$ are elevated in the $\mathrm{OA}$ cartilage (Carpio and Westendorf, 2016), and HDAC4 can promote MMP-13 expression in OA (Song et al., 2015). As a member of class I HDACs, HDAC3 plays an important role in the development of normal cartilage and chondrocyte (Carpio et al., 2016), and also contributes to inflammatory diseases such as carditis (Zhu et al., 2010), spinal injury (Chen S. et al., 2018), and macrophage inflammation (Chen et al., 2012). Regarding arthritis, most studies have focused on the involvement of HDAC3 in leptin-induced arthritis and synovitis in rheumatic arthritis, caused by metabolic dysfunction and autoimmunity dysfunction, respectively (Chang et al., 2015; Angiolilli et al., 2017; Su et al., 2018). Only one article reported elevated HDAC3 was found in the cartilage of OA patients (Meng et al., 2018). Therefore, the role of HDAC3 in OA pathogenesis and exercise therapy requires further investigation.

Nuclear factor-kappaB (NF-kappaB) is a transcription factor that plays an important role in inflammation, especially in OA. NF-kappaB consists of five subunits, and the most common type is the $\mathrm{p} 65 / \mathrm{p} 50$ heterodimer. Upon stimulation by proinflammatory factors, NF-kappaB becomes localized to nucleus and phosphorylated, promoting the expression of MMPs and ADAMTS, which result in degradation of cartilage matrix and OA progression. HDAC3 could regulate NF-kappaB activity. Alteration of acetylation of NF-kappaB by HDAC3 may modulate the transcription activity of NF-kappaB in the relative signal pathway (Ziesche et al., 2013). On the other hand, translocation of HDAC3 to the nucleus could promote NF-kappaB activity (Zhu et al., 2010); and HDAC3 may act as an intra-nuclear molecular switch of the NF-kappaB transcriptional response (Chen and Greene, 2004). However, whether translocation of HDAC3 occurs in chondrocytes and contributes to OA via the HDAC3/NFkappaB pathway is still unclear. ROS, activate the NF-kappaB pathway, promoting inflammation and ECM degradation in OA (Lepetsos and Papavassiliou, 2016). HDAC3 also promotes ROS production in the inflammation response (Zhao et al., 2019). However, whether HDAC3 can regulate ROS production in OA has not been established.

This study aimed (1) to investigate the possible mechanism of physical therapy, which may modulate HDAC3 to prevent OA progression in vivo; (2) to identify the underlying mechanism of HDAC3 in OA in vitro and (3) to investigate the therapeutic effect of the HDAC3 inhibitor RGFP966 in vivo and in vitro (Malvaez et al., 2013; Schmitt et al., 2018). We identified a role for HDAC3 in the pathogenesis of OA, and we found that exercise therapy could attenuate inflammation in OA by modulating the HDAC3/NF-kappaB pathway. Our investigation also showed that RGFP966 exerts an anti-inflammatory effect in OA treatment.

\section{MATERIALS AND METHODS}

\section{Animal Model Construction}

This study was carried out in accordance with the principles of the Basel Declaration and recommendations of U.K. Animals (Scientific Procedures) Act, Ethics Committee of China Medical University. The protocol was approved by the Ethics Committee of China Medical University. During experiments, we adhered to the $3 \mathrm{R}$ rules to ensure that rats were sacrificed comfortably. Adult male Sprague-Dawley (SD) rats (220-240 g; specific-pathogenfree) were purchased from HFK Bioscience, Co., Ltd. (Beijing, China). With a controlled temperature of $22 \pm 2^{\circ} \mathrm{C}$ and $70 \%$ humidity, rats were housed with $12 \mathrm{~h}$ of light/dark cycles and fed with adequate food and drinking water. In order for the rats to adapt to the environment, they were fed for 1 week without any intervention. We anesthetized rats with $2 \%$ pentobarbital sodium by intraperitoneal injection ( $40 \mathrm{mg} / \mathrm{kg}$ ) (Yang et al., 2018c), and shaved the hair around the bilateral knee joints with an electrical razor. Then, $50 \mu \mathrm{l}$ saline containing $0.5 \mathrm{mg}$ MIA $(0.5 \mathrm{mg} / 50 \mu \mathrm{l})$ (Aike Reagent, Co., China) was injected into the intra-articular cavity per knee to construct the OA model according to previous literature (Udo et al., 2016), and $50 \mu \mathrm{l}$ saline was injected per knee for the control group. The OA model was induced by intraarticular injection using a $50 \mu \mathrm{lmicro-syringe} \mathrm{through} \mathrm{the} \mathrm{lateral}$ infrapatellar area into the distal femoral condylar space in an excessive knee flexion position.

\section{Exercise Protocol}

We used rat-specific treadmill (ZH-PT, Zhongshidichuang Science \& Technology Development, Co., Ltd., Beijing, China) for the experiments. Several generators were applied to the grid at the end of every lane, which could provide acoustic stimulation $(80 \mathrm{~dB})$, light stimulation $(300 \mathrm{~lx})$ and electric stimulation $(100 \mathrm{~Hz}, 0.18 \mathrm{~mA})$ to encourage rats when they failed to run on the treadmill. Firstly, rats were forced to run at a speed of 10 $\mathrm{m} / \mathrm{min}$ for $10 \mathrm{~min} /$ day for 5 days to adapt to treadmill exercise. 
All rats $(n=50)$ were randomly divided into four groups: control group (CG); OA model group (OAG); MIA + low-intensity exercise group (OAL); MIA + moderate-intensity exercise group $(\mathrm{OAM})$; and MIA + high-intensity exercise group (OAH).

\section{Formal Treadmill Exercise}

We set the speed for the OAH group at $26 \mathrm{~m} / \mathrm{min}, 60 \mathrm{~min} /$ day, 5 days/week for 4 weeks; the OAM group at $18 \mathrm{~m} / \mathrm{min}$, $60 \mathrm{~min} /$ day, 5 days/week for 4 weeks and the OAL group at $12 \mathrm{~m} / \mathrm{min}, 60 \mathrm{~min} /$ day, 5 days/week for 4 weeks. The treadmill incline was set as 5\% (Sekiya et al., 2009) (Figure 1A). The OAL, OAM, and OAH groups began to run after MIAinjection for $24 \mathrm{~h}$. The $\mathrm{CG}$ and $\mathrm{OAG}$ were kept in cages without any intervention. Body weight was recorded at regular intervals (Figure 1B). During the experimental period, rats that failed to run despite application of combined stimulation were defined as drop-outs and excluded from the experimental groups. Meanwhile, observation was required to ensure smooth execution of experiments, and the rats' completion rate was calculated every week (Saito et al., 2017) (Figure 1C). Rats were sacrificed at the end of the experimental period.

\section{RGFP966-Injection in the OA Model}

All SD rats $(n=40)$ were divided into four groups: CG, OAG, OA + RGFP966 group and RGFP966 group. The concentration of RGFP966 (MCE, HY13909) was $50 \mu \mathrm{M}$ per knee. RGFP966 was first dissolved with DMSO and then diluted with saline (concentration of DMSO <2\%). $50 \mu \mathrm{M}$ RGFP966 was administered by intra-articular injection per knee, 3 times/week for 4 weeks (Jia et al., 2016). CG was injected with same volume of saline; OAG was injected with $50 \mu \mathrm{l}$ saline containing $0.5 \mathrm{mg}$ MIA $(0.5 \mathrm{mg} / 50 \mu \mathrm{l})$; OA + RGFP966 group was injected with RGFP966 after MIA injection for 24 h; RGFP966 group was only injected with RGFP966. The method of OA model construction and articular injection was the same as above mentioned. The rats were sacrificed after 4 weeks.

\section{Cell Culture}

Primary rat chondrocytes were isolated from the knee joints of 4 week-old adult male Sprague-Dawley rats (100-150 g; specific pathogen-free). Primary rat chondrocytes were obtained by a two-steps enzymatic digestion. The cartilage was cut into $1 \mathrm{~mm}^{2}$ pieces followed by washing with PBS. Cartilage pieces were then digested with pronase $\mathrm{K}$ ( $4 \mathrm{mg} / \mathrm{ml}$, Roche, Basel, Switzerland) for $1 \mathrm{~h}$ at $37^{\circ} \mathrm{C}$ and collagenase $\mathrm{D}(1.6 \mathrm{mg} / \mathrm{ml}$, Roche) for $1 \mathrm{~h}$ at $37^{\circ} \mathrm{C}$. Gentle mechanical vibration was applied in the whole digestion. Cell suspensions were centrifuged at $800 \mathrm{rpm}$ for $5 \mathrm{~min}$, and resuspended in PBS. The suspensions were filtered through a $100 \mu \mathrm{m}$ mesh to exclude micro-tissue pieces. Undigested cartilage pieces were then treated with collagenase D according to the protocol mentioned above until remained cartilage pieces disappeared. Primary rat chondrocytes were then transferred into $25 \mathrm{~cm}^{2}$ culture flasks at a density of $5 \times 10^{6}$ cells, and cultured with DMEM with low D-glucose (Hyclone, South Logan, UT, United States) supplemented with 10\% FBS (Bioind, China) and $1 \%$ penicillin and streptomycin (Hyclone) at $37^{\circ} \mathrm{C}$ in a $5 \% \mathrm{CO}_{2}$ incubator. After attachment of chondrocytes, culture medium was replaced. Chondrocytes were split in a 1:3 ratio when cell confluence reached $70 \%$. Immunocytochemical staining of collagen II was used to identify the purity of chondrocytes. To guarantee the chondrocyte phenotype and accuracy of the subsequent experiments, primary rat chondrocytes at passage 3 were used. Cells were stimulated with IL-1 $\beta$ (R\&D, Minneapolis, $\mathrm{MN}$, United States) at a concentration of $20 \mathrm{ng} / \mathrm{ml}$ for $24 \mathrm{~h}$, to obtain OA-like chondrocytes. To determine the role of RGFP966 in inflammation response in chondrocyte, chondrocytes were

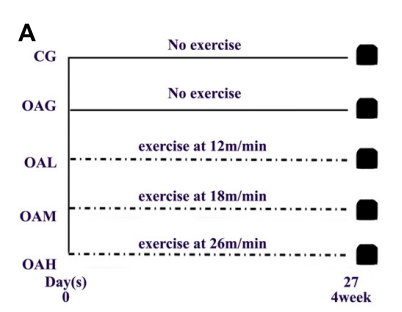

B

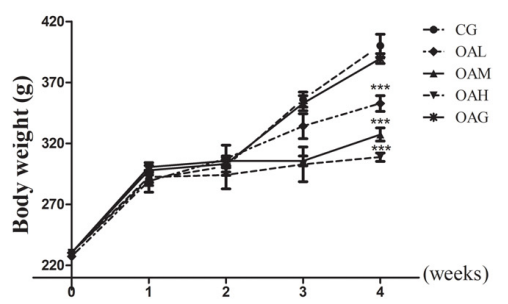

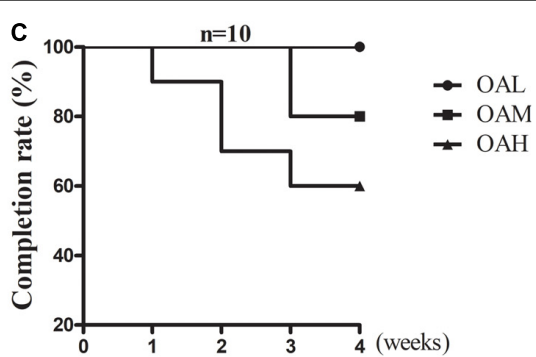

FIGURE 1 | Protocols of different intensities of treadmill exercise on OA rats and completion rate, weight change of OA rats. (A) Different intensities of treadmill exercise on OA rats; speeds of 12, 18, and $26 \mathrm{~m} / \mathrm{min}$ represent OAL, OAM and OAH respectively, and all groups were sacrificed after 4 weeks. (B) Weight change of rats in different groups. There was significant difference between exercise group and $\mathrm{CG}, \mathrm{OAG}$, whereas difference among OAL, OAM, and OAH was not significant; ${ }^{* * *} p<0.001 ; n=6-10$ rats for each group, means with SEM. (C) Completion rate of rats of different intensities of treadmill exercise. The completion was decreased along with increased intensity of treadmill exercise. 
incubated with IL-1 $\beta(20 \mathrm{ng} / \mathrm{ml})$ and RGFP966 (10 $\mu \mathrm{M})$ simultaneously. Then, the chondrocytes were collected after $24 \mathrm{~h}$ incubation.

\section{X-Ray Imaging Observation}

Knee joint images were captured by X-ray (MX-20, Faxitron X-Ray, Corp., Lincolnshire, IL, United States). Rats were anesthetized with pentobarbital sodium by intraperitoneal injection $(40 \mathrm{mg} / \mathrm{kg})$, and immobilized in the supine position with the bilateral ankles fixed on the tray by adhesive tape. The lens was focused on the rats' knee with an appropriate focal length and the exposure time was set at $5 \mathrm{~min}$ to ensure clear images. The degree of OA was evaluated by imaging manifestation, including narrowing of joint space, and calcification changes of articular surface according to the X-ray image score system in previous literature (Wei et al., 2017). All images were measured by two investigators in a blinded manner.

\section{Histological Examination}

Knee joints with intact articular capsules were harvested and fixed in $4 \%$ paraformaldehyde for 7 days at room temperature, followed by decalcification in 10\% EDTA solution for 28 days at $37^{\circ} \mathrm{C}$. EDTA solution refreshed every 3 days. Specimens were dehydrated in graded alcohol, made transparent in xylene and embedded in paraffin wax. Specimens were sectioned at $4.5 \mathrm{~mm}$ in the sagittal position with intact epiphyseal line in both the tibial and femur portions, and stained with hematoxylin and eosin (H\&E) and Toluidine blue O. Histological sections were visualized using an Olympus BX53 microscope (Olympus, Tokyo, Japan). The degree of cartilage damage of the knee joints was evaluated according to OARSI scores and Mankin scores (Custers et al., 2007; Gerwin et al., 2010; Lee et al., 2011). The range of OARSI and Mankin scores was $0-24$ and $0-14$ points, respectively. Femoral and tibial cartilages were evaluated separately. OA was scored by two individuals in a blinded manner, using a validated histologic scoring system.

\section{Immunostaining}

Sections were deparaffinized in xylene and graded alcohol and washed using cold PBS. Subsequent steps were performed in humidified conditions. Enzymatic antigen retrieval (AR0026, Boster, Biological Technology, Pleasanton, CA, United States) was used to repair antigens at $37^{\circ} \mathrm{C}$ for $30 \mathrm{~min}$. After that, endogenous peroxidase activity was eliminated by $3 \%$ $\mathrm{H}_{2} \mathrm{O}_{2}$ for $30 \mathrm{~min}$ at room temperature followed by washing there times in PBS; 5\% goat serum was used to block non-specific antigens. Next, sections were incubated with rabbit polyclonal anti-collagen II antibody (1:50, Abcam, ab34712), rabbit polyclonal anti-MMP-13 antibody (1:50, Abcam, ab39012), rabbit polyclonal anti-ADAMTS5 antibody (1:50, Bioss, bs3573R), rabbit polyclonal anti-NF-kappaB P65 antibody (1:50, Proteintech, 10745-1-AP) and rabbit polyclonal antiHDAC3 antibody (1:50, Proteintech, 10255-1-AP) at $4^{\circ} \mathrm{C}$ overnight. After extensive washing there times with PBS, biotinylated secondary antibodies were added to the sections at room temperature for $30 \mathrm{~min}$, followed by washing three times in PBS; sections were then incubated with streptavidin/horseradish peroxidase (S-A/HRP) (Zhongshan Goldenbridge Biotechnology, Co., China) at room temperature for $30 \mathrm{~min}$ according to the manufacturer's protocol. Sections were visualized with diaminobenzidine (DAB) staining for $1 \mathrm{~min}$ and counterstained with hematoxylin for $5 \mathrm{~min}$. Sections were dehydrated with graded alcohol and xylene, and sealed with neural gums. Images of sections were captured with optical microscopy (Eclipse Ci, Nikon, Japan) and optical density was measured with image analysis software Image-Pro Plus, version 6.0 (Media Cybernetics, Inc., Rockville, MD, United States). Rabbit IgG as the primary antibody was used as a negative control, and other steps were similar to the protocol mentioned above (Supplementary Figure S4). Three images of each slide were captured at a $400 \times$ magnification, and the average optical density represented relative expression of protein; NFkappaB was calculated by percentage of positively-stained cell (Zhang et al., 2019).

\section{Western Blot Analysis}

Cells and rat cartilage were lysed in RIPA buffer $(25 \mathrm{mM}$ Tris$\mathrm{HCl} \mathrm{pH} \mathrm{7.6,} 150 \mathrm{mM} \mathrm{NaCl}, 1 \% \mathrm{NP}-40,1 \%$ sodium deoxycholate, $0.1 \%$ SDS) with $1 \mathrm{mM}$ PMSF (Beyotime, ST506). Lysates were centrifuged at $14000 \mathrm{~g} / \mathrm{min}$ for $5 \mathrm{~min}$ at $4^{\circ} \mathrm{C}$ and supernatants were collected. Proteins in the cytoplasm and nucleus were isolated by using a Cytoplasmic and Nuclear Protein Extraction Kit (78833, Thermo Fisher, United States), according to the manufacturer's instructions and previous study (Huang et al., 2014). Protein concentration was measured by the bicinchoninic acid assay (Beyotime, P0010). Equal amounts of protein (40 mg) were separated by sodium dodecyl sulfate polyacrylamide gel electrophoresis, and transferred to polyvinylidene difluoride membranes (Millipore, Bedford, MA, United States). Membranes were then blocked with $5 \%$ non-fat milk (w/v) for $2 \mathrm{~h}$ at room temperature followed by incubation with primary antibodies overnight at $4^{\circ} \mathrm{C}$. The following antibodies were used: rabbit polyclonal anti-HDAC3 antibody (1:1000, CST, 3949S), rabbit polyclonal anti-collagen II antibody (1:10000, Abcam, ab34712), rabbit polyclonal anti-MMP-13 antibody (1:2000, Abcam, ab39012), mouse monoclonal anti-GAPDH antibody (1:4000, Proteintech, 60004-1-Ig), rabbit polyclonal anti- NF-kappaB p65 antibody (1:4000, Proteintech, 10745-1AP), rabbit polyclonal anti- $\beta$-actin (1:1000, Proteintech, 205361-AP), rabbit polyclonal anti-H3 antibody (1:4000, Proteintech, 17168-1-AP). After washing three times with Tris-buffered saline with $0.1 \%$ Tween-20 (TBST), the membranes were incubated with horseradish peroxidase-conjugated secondary antibodies (Beyotime, China) for $2 \mathrm{~h}$ at room temperature. Membranes were then washed with TBST three times; bands were detected by enhanced chemiluminescence (Millipore), and quantified using Image J software (National Institutes of Health). $\beta$-Actin and $\mathrm{H} 3$ were used as the cytoplasmic and nuclear internal controls, respectively (Supplementary Figure S6).

\section{Co-immunoprecipitation (Co-IP) Assay}

Chondrocytes were lysed in IP RIPA (Beyotime, P0013) with Protease inhibitor cocktail (Beyotime, P1005). Lysates were centrifuged at $14000 \mathrm{~g} / \mathrm{min}$ for $5 \mathrm{~min}$ at $4^{\circ} \mathrm{C}$ and supernatants 
were collected. $500 \mu \mathrm{g}$ protein lysates were incubated with $30 \mu \mathrm{l}$ protein A/G PLUS-Agarose beads (SantaCruz sc-2003) and $8 \mu \mathrm{l}$ anti-NF-kappaB p65 antibody (Proteintech, 10745-1-AP) at $4^{\circ} \mathrm{C}$ overnight. Next day, the beads were washed three times with lysis buffer and collected at $1500 \mathrm{rpm}$ at $4^{\circ} \mathrm{C}$ for $5 \mathrm{~min}$. The beads mixed with $30 \mu 12 \times$ loading buffer for the SDS-PAGE electrophoresis.

\section{Quantitative Reverse Transcription Polymerase Chain Reaction (qRT-PCR)}

Total RNA was isolated from primary rat chondrocytes and rat model cartilage tissue using RNAiso Plus (Takara, Shiga, Japan) and $1 \mu \mathrm{g}$ RNA was reverse transcribed into cDNA using PrimeScript RT reagent Kit with gDNA Eraser (Takara) according to the manufacturer's instructions. The polymerase chain reaction (PCR) reaction was completed with a SYBR Green PCR kit (Takara) using the Applied Biosystems 7500 Real-Time PCR System in triplicate per primer and per sample. Operation conditions were: $95^{\circ} \mathrm{C}$ for $30 \mathrm{~s}, 95^{\circ} \mathrm{C}$ for $5 \mathrm{~s}, 40$ cycles of $60^{\circ} \mathrm{C}$

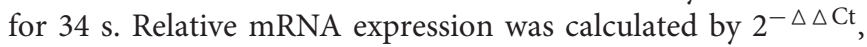
which represented the fold-change with $\beta$-actin as the internal control. The following primers were designed by and purchased from Sangon, China (Table 1).

\section{Cell Counting Kit-8 (CCK-8) Test}

Primary rat chondrocytes were seeded in two 96-well plates $\left(5 \times 10^{3}\right.$ cells per well) and cultured for $1-2$ days at $37^{\circ} \mathrm{C}$ with DMEM containing 10\% FBS until cell confluency reached $70 \%$. One plate was then used for culture with serum-free DMEM containing graded concentration of RGFP966 (0, 0.3, 0.6, 1.2, 2.5, 5 , and $10 \mu \mathrm{M}$ ) for $24 \mathrm{~h}$ at $37^{\circ} \mathrm{C}$. The other 96 -well plate was used for culture with serum-free DMEM containing $10 \mu \mathrm{M}$ RGFP966 cultured at $37^{\circ} \mathrm{C}$ for varying times $(0,6,12,24,36$, and $48 \mathrm{~h})$. After RGFP966 treatment, $10 \mu$ l CCK-8 (Beyotime, C0042) dissolved in $90 \mu \mathrm{l}$ serum-free DMEM was added to each well followed by incubation for $2 \mathrm{~h}$ at $37^{\circ} \mathrm{C}$. The absorbance value of each well was measured at wave length of $450 \mathrm{~nm}$ using a spectrophotometer (Synergy H1, BioTek, United States). The entire experiment was repeated five times.

\section{Cellular ROS Production}

Chondrocytes were seeded and cultured in six-well plates at a density of $1 \times 10^{5}$ per well. When confluency of chondrocyte reached $80 \%$, chondrocytes were stimulated with IL-1 $\beta(10 \mathrm{ng} / \mathrm{ml})$ and/or RGFP966 (10 $\mu \mathrm{M})$ with serumfree DMEM for $24 \mathrm{~h}$. ROS production was measured by flow cytometry with dichloro-dihydrofluorescein diacetate (DCFH-DA) (S0033, Beyotime). Chondrocytes were harvested and incubated with $10 \mathrm{mM}$ DCFH-DA for $30 \mathrm{~min}$ at $37^{\circ} \mathrm{C}$ in the dark, and then washed three times in PBS. The negative control group included serum-free DMEM with no DCFH-DA (Supplementary Figure S5). Finally, 10000 events were analyzed with a FACSCalibur flow cytometer (BD Biosciences, San Diego, CA, United States) with $488 \mathrm{~nm}$ excitation and $525 \mathrm{~nm}$ emission wavelengths (Jeong et al., 2019). The mean value of ROS intensity in each
TABLE 1 | Sequences of primers used for real-time PCR.

\begin{tabular}{ll}
\hline Gene symbol & Sequence \\
\hline HDAC3 forward & $5^{\prime}$-TTGAAGATGCTGAACCATGC-3' \\
HDAC3 reverse & $3^{\prime}$-TGGCCTGCTGTAGTTCTCCT-5' \\
ADAMTS-5 forward & $5^{\prime}$-TCCTCTTGGTGGCTGACTCT-3' \\
ADAMTS-5 reverse & $3^{\prime}$-GGATGTGGTTCTCGATGCTT-5' \\
MMP-13 forward & $5^{\prime}$-TGGTCCAGGAGATGAAGACC-3' \\
MMP-13 reverse & $3^{\prime}$-GTGCAGACGCCAGAAGAATC-5' \\
Collagen II forward & $5^{\prime}$-ACGCTCAAGTCGCTGAACAACC-3' \\
Collagen II reverse & $3^{\prime}$-ATCCAGTAGTCTCCGCTCTTCCAC-5' \\
NF-kappaB p65 forward & $5^{\prime}$-GGCTTCTATGAGGCTGAACTCTGC-3' \\
NF-kappaB p65 reverse & $3^{\prime}$-CTTGCTCCAGGTCTCGCTTCTTC-5' \\
$\beta$-Actin forward & $5^{\prime}$-CACCCGCGAGTACAACCTTC-3' \\
$\beta$-Actin reverse & $3^{\prime}$-CCACACTACCACCCATACCC-5' \\
\hline
\end{tabular}

group was used to analysis difference by One-way analysis of variance.

\section{Statistical Analysis}

Results are represented as mean \pm SEM using GraphPad Prism 5. The Shapiro-Wilk and Levene tests were used to examine the normality and homogeneity of the results, respectively. One-way analysis of variance was used for the parametric analysis. Nonparametric data were analyzed by the Kruskal-Wallis $H$-test. $p$-Values of $<0.05$ were considered statistically significant.

\section{RESULTS}

\section{Weight Change and Completion Rate of Rats in the Treadmill Exercise Program}

As well-established, weight load can affect cartilage development; accordingly overload was also a cause of OA. We therefore detected weight changes of the rats in the program (Ernest and Kondrashov, 2018). Compared with the CG, the average weight of rats in the different exercise groups was significantly lower. Meanwhile, there were no differences in weight between the $\mathrm{OAM}$ and $\mathrm{OAH}$ groups, although OA symptoms were fewer in the OAM group compared to the OAH group (Figure 1B). We suggest that weight changes did not affect our experiments. According to the literature (Saito et al., 2017), we knew that not all rats could complete the exercise program due to intolerant intensity, especially in the OAH group. We found that all rats in the OAL group were able to complete the program; dropout began at 3 week, and $80 \%$ of rats completed the program in the OAM group. Drop-out began at 1 week and $60 \%$ of rats completed the program in the OAH group (Figure 1C).

\section{Histological Changes of OA Affected by Treadmill Exercise}

We constructed the most suitable OA rat model successfully, according to our previous study (Yang et al., 2017), and then explored the effect of treadmill exercise on OA. We observed histological changes in the articular joints of OA rats in conditions of different exercise intensities. In macroscopic and 


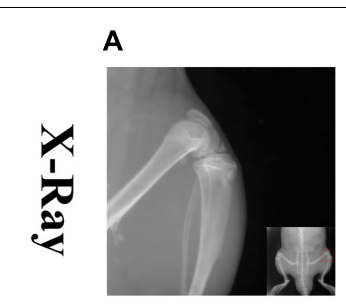

CG

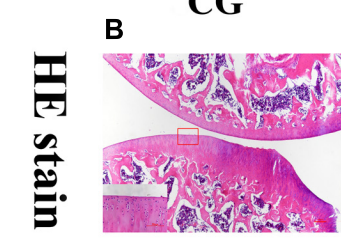

CG

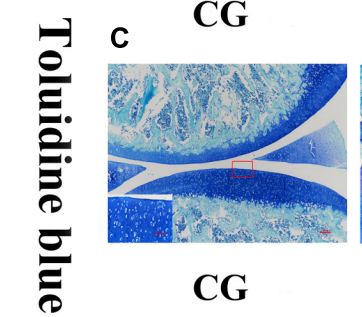

D

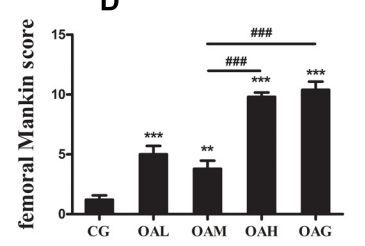

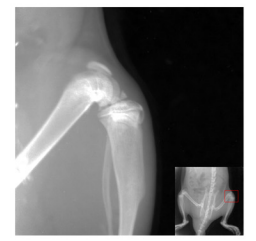

OAL

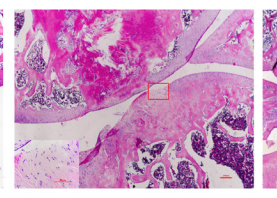

OAL

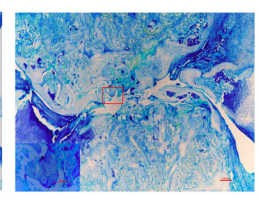

OAL

E

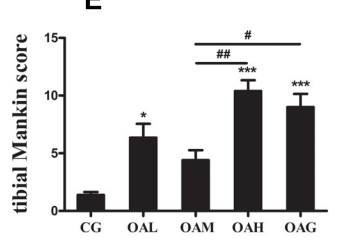

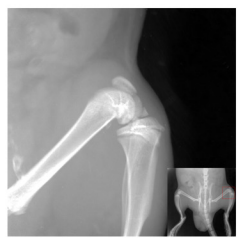

OAM

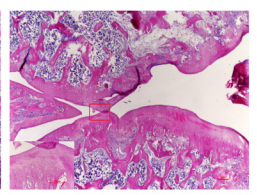

OAM

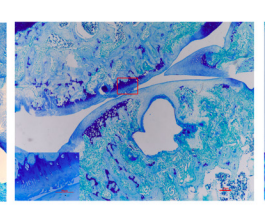

OAM

F

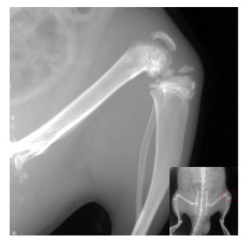

OAH

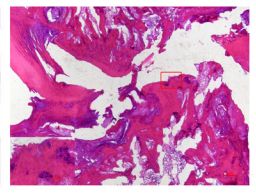

OAH

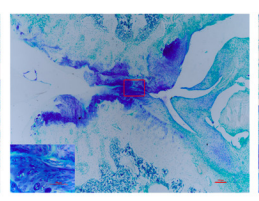

OAH

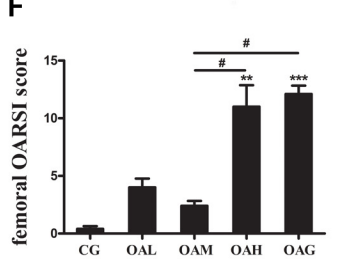

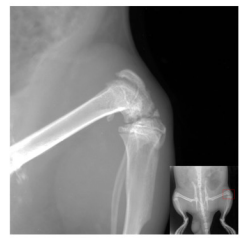

OAG

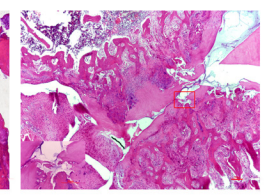

OAG

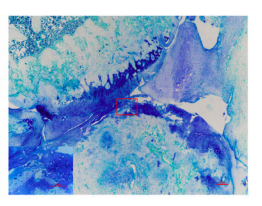

OAG

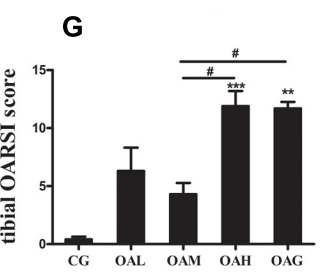

FIGURE 2 | Histological changes of the articular joints of OA rats treated with different intensities of treadmill exercise. (A) X-ray image of articular joints of OA rats. Articular damage was severe in OAH and OAG, whereas the appearance was light in OAM. (B) H\&E image of articular joints of OA rats. Articular surface was disappeared in $\mathrm{OAH}$ and $\mathrm{OAG}$, whereas articular surface refreshed in OAM. (C) Toluidine blue $\mathrm{O}$ image of articular joints of OA rats. Cartilage erosion and staining reduction were in $\mathrm{OAH}$ and OAG, whereas compensatory regeneration of cartilage was in OAM. (D) Femoral Mankin score for articular joints. Femoral Mankin score of CG was lowest; OAM was lower than that of OAH and OAG. (E) Tibial Mankin score for articular joints. Tibial Mankin score of CG was lowest; OAM was lower than that of OAH and OAG. (F) Femoral OARSI score for articular joints. Femoral OARSI score of CG was lowest; OAM was lower than that of OAH and OAG. (G) Tibial OARSI score for articular joints. Femoral OARSI score of CG was lowest; OAM was lower than that of OAH and OAG; ${ }^{* * *} p<0.001$; ${ }^{* *} p<0.01$; ${ }^{*} p<0.05$ vs. CG, ${ }^{\# \# \# ~} p$ 0.001; ${ }^{\# \#} p<0.01 ;{ }^{\#} p<0.05$ vs. OAM; $n=5$ rats for each group, means with SEM.

imaging observations, we found cartilage damage and synovitis in the OAH group, similar to the OAG (Figure 2A), whereas the OAM was shown to alleviate OA symptoms. In X-ray examinations, imaging symptoms and score in the CG and OAM were lower than the OAG and OAH (Supplementary Figure S1). As for $\mathrm{H} \& \mathrm{E}$ and Toluidine blue $\mathrm{O}$ staining, we found cartilage surface erosion and chondrocyte death in the OAH group; nevertheless, OAM could refresh the cartilage surface and relieve chondrocyte death (Figures 2B,C). Meanwhile, the OARSI and Mankin scores of tibial and femoral cartilage were also lower in the OAM group than the OAH and OAG group (Figures 2D-G).

\section{The Expression of Relative Inflammatory Proteins/Genes in OA Treated With Treadmill Exercise}

We identified the expression of OA-relative proteins treated with different intensities of treadmill exercise by immunohistochemistry (IHC) and western blotting. The expression of collagen II protein was increased in the OAM group compared to the $\mathrm{OAH}$ group or OAG (Figures $\mathbf{3 A}, \mathbf{C}, \mathbf{4 A}, \mathbf{B}$ ), whereas MMP-13 and ADAMTS-5 were decreased in the OAM group compared to the OAH group or OAG (Figures $4 A, C, D$ ). We also detected NF-kappaB expression in the cytoplasm and nucleus; nuclear expression of NF-kappaB was significantly lower in the OAM group than the $\mathrm{OAH}$ group and $\mathrm{OAG}$ according to IHC, whereas nuclear expression of NF-kappaB was lower in the OAM group compared to the OAG according to Western blotting; expression of NF-kappaB in the cytoplasm was also significantly decreased in the OAM group compared to the OAH group or OAG (Figures $\mathbf{3 A}, \mathbf{B}, \mathbf{D}, \mathbf{F}, \mathbf{4 A}, \mathbf{E}$ ). We found that expression of HDAC3 protein (both in the nucleus and cytoplasm) was significantly decreased in the OAM group compared to OAG (Figures $\mathbf{3 A}, \mathbf{B}, \mathbf{E}, \mathbf{G}$ ), and that the total HDAC3 protein was decreased in the OAM compared to OAH group and OAG (Figures 4A,F). We also detected mRNA 


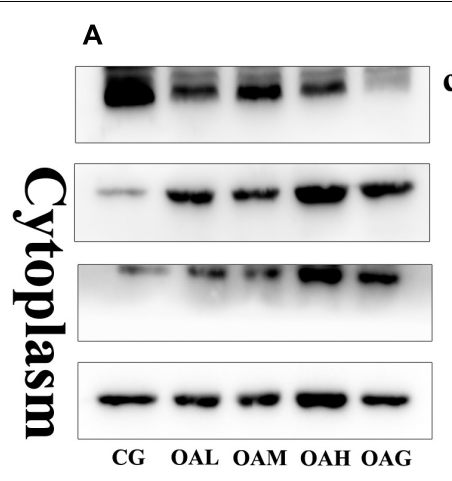

\section{collagenII $142 \mathrm{kD}$

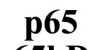 \\ $65 \mathrm{kD}$ \\ HDAC3 \\ 49kD \\ Actin \\ 46kD}

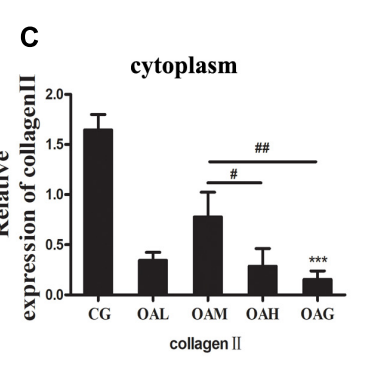

\section{D}

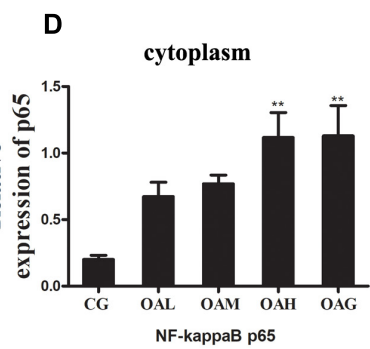

B

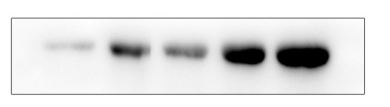

p65

$65 \mathrm{kD}$

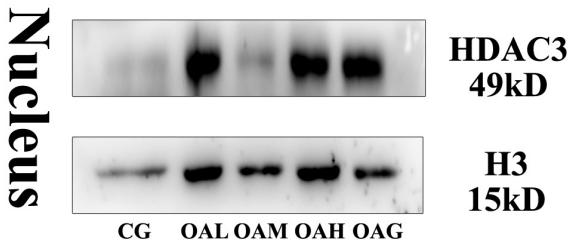

CG OAL OAM OAH OAG
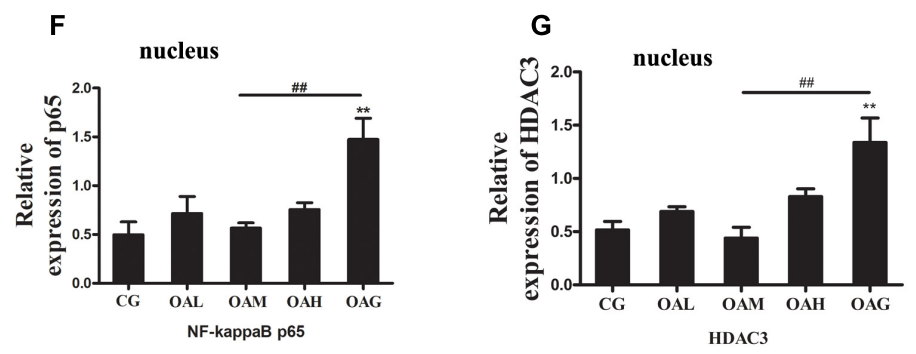

FIGURE 3 | Osteoarthritis rats were treated with different intensities of treadmill exercise. Cytoplasmic and nuclear proteins were extracted from the articular cartilage of rats. Western blotting was performed to measure relative inflammatory proteins and HDAC3 in OA. The data was obtained in three separate experiments with $\beta$-actin as a cytoplasmic internal control, and $\mathrm{H} 3$ as a nuclear internal control. (A,C) Expression of collagen II in cytoplasm in CG was highest, and expression of collagen II in cytoplasm in OAM was higher than OAG and OAH. (A,D) Expression of NF-kappaB in cytoplasm in OAH and OAG was higher than CG, and the difference among OAM and OAH, OAG was not significant. (A,E) Expression of HDAC3 in cytoplasm in CG was lowest, and expression of HDAC3 in cytoplasm in OAM was lower than OAH and OAG. (B,F) Expression of NF-kappaB in nucleus in OAG was higher than CG and OAM. (B,G) Expression of HDAC3 in the nucleus in OAG was higher than CG and OAM; ${ }^{* * *} p<0.001 ;{ }^{* *} p<0.01 ;{ }^{*} p<0.05$ vs. CG, \#\# $p<0.01 ; \# p<0.05$ vs. OAM; $n=3$ rats for each group, means with SEM.

changes by real-time PCR; collagen II mRNA level increased in the OAM group compared to the OAH group or OAG (Figure 4G); MMP-13 mRNA level were lower in the OAM group than the OAG (Figure 4H); ADAMTS5, HDAC3 and NF-kappaB mRNA levels were greater in the OAM group compared to the OAH group and OAG (Figures 4I-K).

\section{Histological Changes and Expression of Relative Proteins of OA Affected by RGFP966}

To confirm the role of HDAC3 in OA in vivo, we treated OA rats with HDAC3-specific inhibitor RGFP966 by intra-articular injection for 4 weeks. In $\mathrm{H} \& \mathrm{E}$ and Toluidine blue $\mathrm{O}$ staining, the level of cartilage surface damage and chondrocyte death in the OAG + RGFP966 group was lower than the OAG (Figures 5A,B), which was also represented by OARSI and Mankin scores of femoral and tibial cartilage (Figures 5C-F).
We then detected the expression of OA-relative proteins in OA with intra-articular injection with RGFP966 by IHC. Collagen II in the OAG significantly was lower than the CG and OAG + RGFP966 group (Figures 6A,B), whereas MMP-13, percentage of NF-kappaB cells and HDAC3 in the OAG were significantly greater compared with the $\mathrm{CG}$ and OAG + RGFP966 group (Figures 6A,C-E). We therefore concluded that RGFP966 had a protective effect on OA in vivo.

\section{Isolation and Identification of Primary Rat Chondrocytes}

To ensure accuracy of the subsequent experiments, we identified primary rat chondrocytes isolated from male rat knee cartilage. Under microscopic examination, the shape of normal chondrocyte was polygonal (Figure 7A). We also identified chondrocytes through IHC using the collagen II antibody (Figure 7B). 

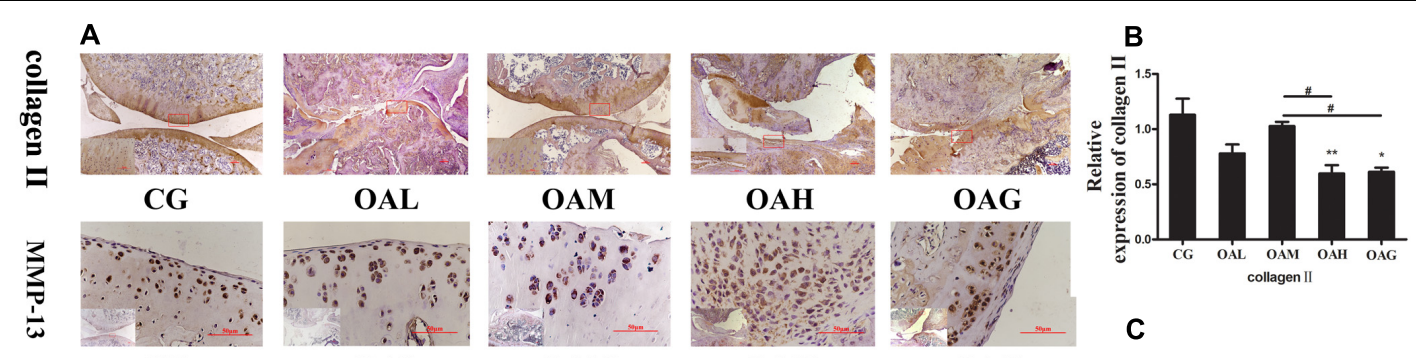

OAL

OAM
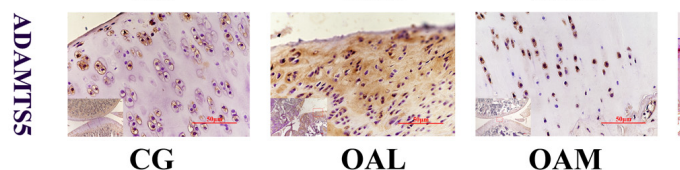

OAH

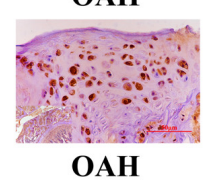

OAG
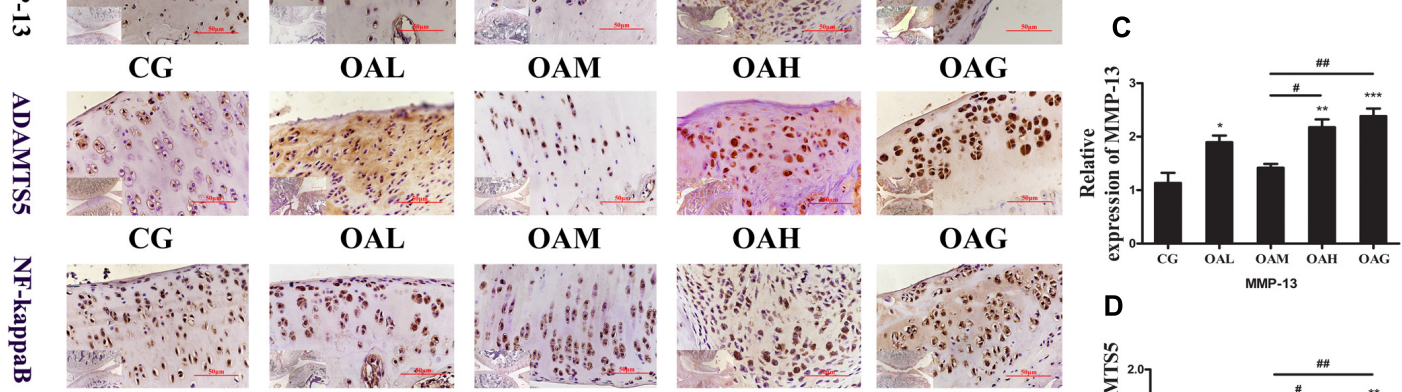

OAL
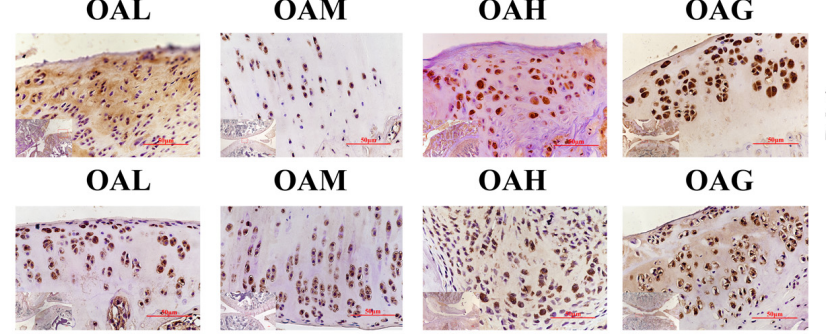

OAL

OAM
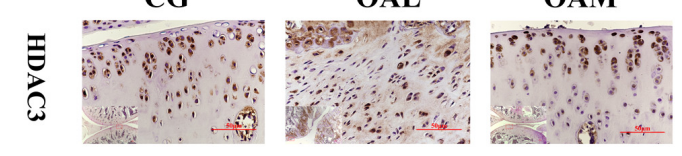

OAH
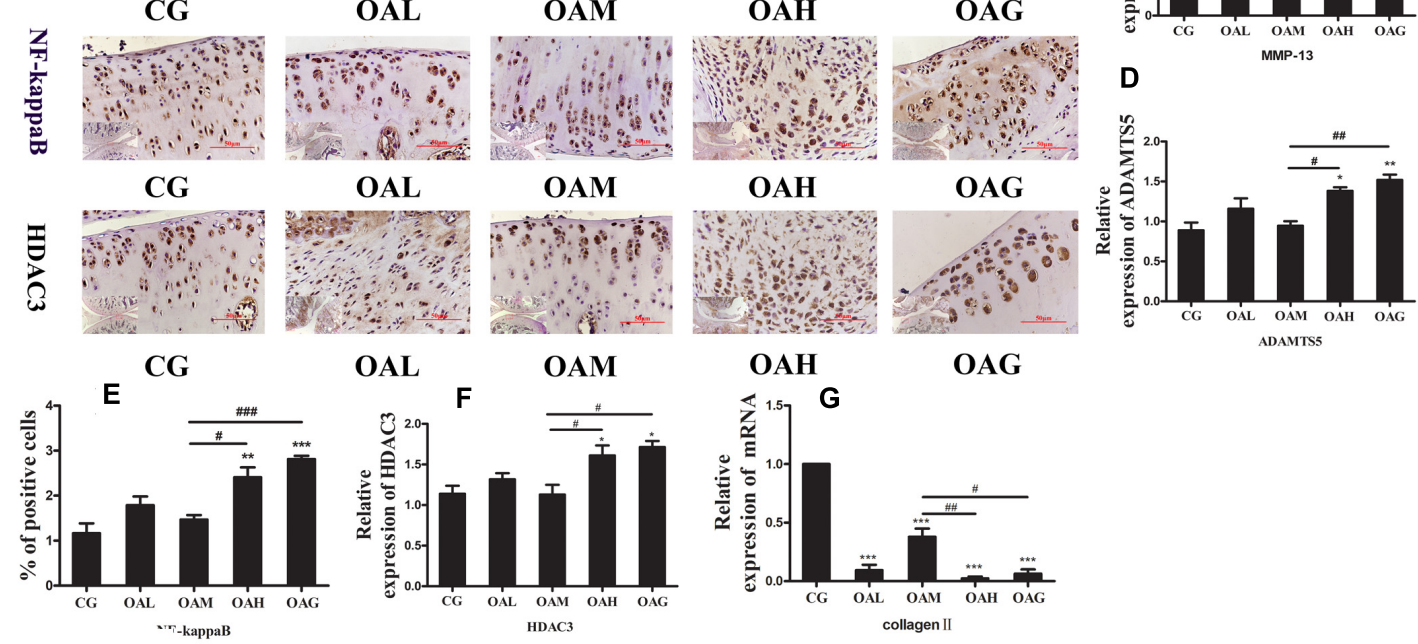

OAL
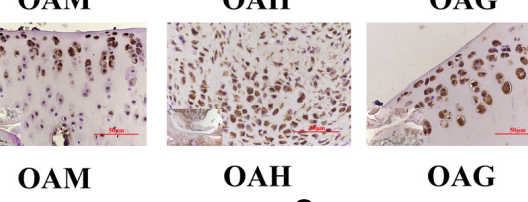

OAG
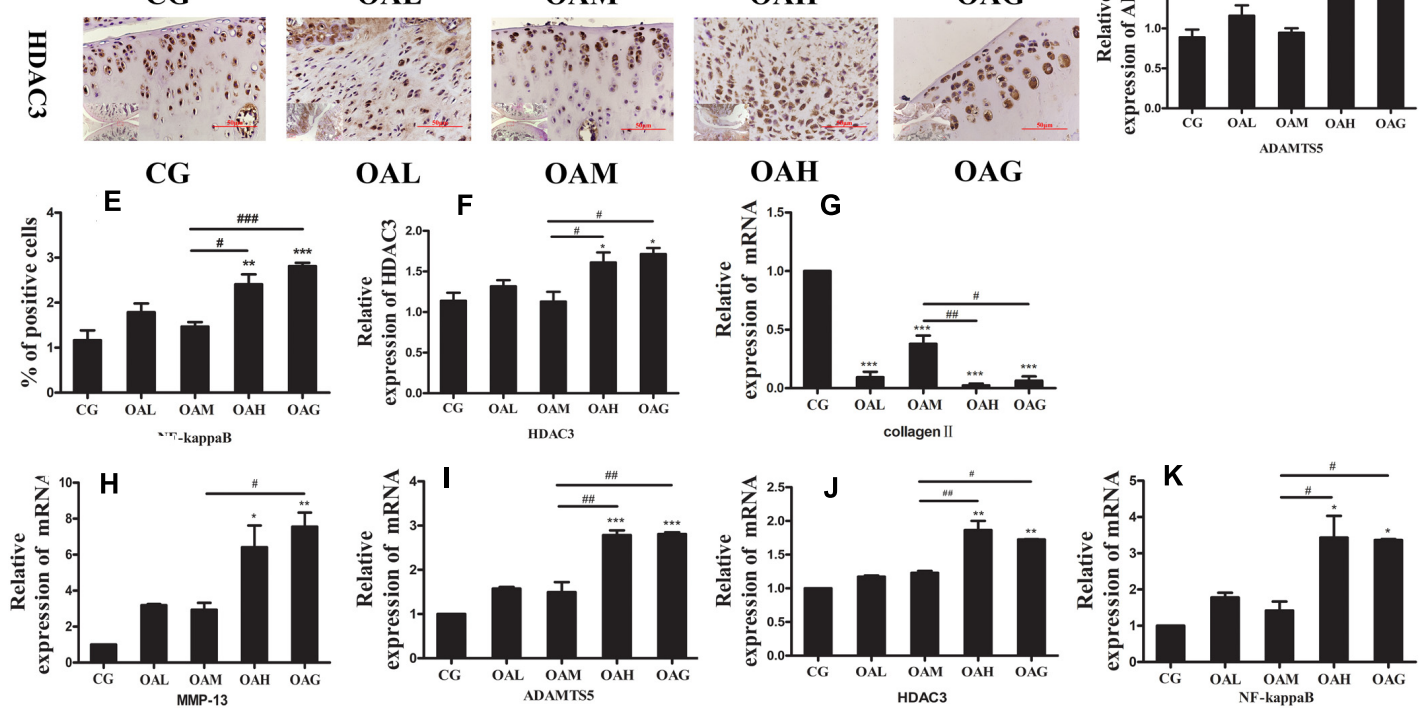

FIGURE 4 | Osteoarthritis rats were treated with different intensities of treadmill exercise. Real-time PCR and immunohistochemistry (IHC) were performed to measure relative inflammatory genes/proteins in OA. (A,B) Expression of collagen II in CG and OAM were higher than OAH and OAG by IHC. (A,C) Expression of MMP-13 in CG and OAM were lower than OAH and OAG by IHC. (A,D) Expression of ADAMTS5 in CG and OAM were lower than OAH and OAG by IHC. (A,E) The percentage of NF-kappaB positive cell in CG and OAM were lower than OAH and OAG by IHC. (A,F) Expression of HDAC3 in CG and OAM were lower than OAH and OAG by IHC. (A,G) mRNA of collagen II in CG and OAM were higher than OAH and OAG by real-time PCR analysis. (A,H) mRNA of MMP-13 in CG and OAM were lower than OAG and mRNA of MMP-13 in CG was lower than OAH by real-time PCR analysis. (A,I) mRNA of ADAMTS5 in CG and OAM were lower than OAH and OAG by real-time PCR analysis. (A,J) mRNA of HDAC3 in CG and OAM were lower than OAH and OAG by real-time PCR analysis. (A,K) mRNA of NF-kappaB in CG and OAM were lower than OAH and OAG by real-time PCR analysis; ${ }^{* * *} p<0.001 ; *^{* *} p<0.01 ;{ }^{*} p<0.05$ vs. CG, ${ }^{* \#} p<0.001$; ${ }^{\# \#} p<0.01$; ${ }^{*} p<0.05$ vs. $\mathrm{OAM} ; n=3$ rats for each group in $\mathrm{HC}$ and $n=6$ rats for each group in real-time PCR, means with SEM.

\section{The Effect of HDAC3 Inhibitor RGFP966 on Chondrocyte Viability}

There has been little reported evidence about the effect of RGFP966 on chondrocyte. To identify the most suitable concentration of RGFP966, we firstly identified the changes in chondrocyte viability stimulated with different RGFP966 concentrations. We established a concentration range from 1 to $10 \mu \mathrm{M}$ according to the literature (Leus et al., 2016; Correa et al., 2017). We found that RGFP966 can promote chondrocyte viability in a dose-dependent manner, and that $10 \mu \mathrm{M}$ was the most suitable concentration (Figure 7C). We then investigated chondrocyte viability stimulated with $10 \mu \mathrm{M}$ RGFP966 with different durations. We found that RGFP966 also promoted chondrocyte viability in a time-dependent manner and that $24 \mathrm{~h}$ was the most suitable time (Figure 7D).

\section{RGFP966 Attenuated Nuclear Location of NF-KappaB Mediated by HDAC3}

NF-kappaB is an important transcription factor that plays a crucial role in OA. More NF-kappaB in the nucleus means 


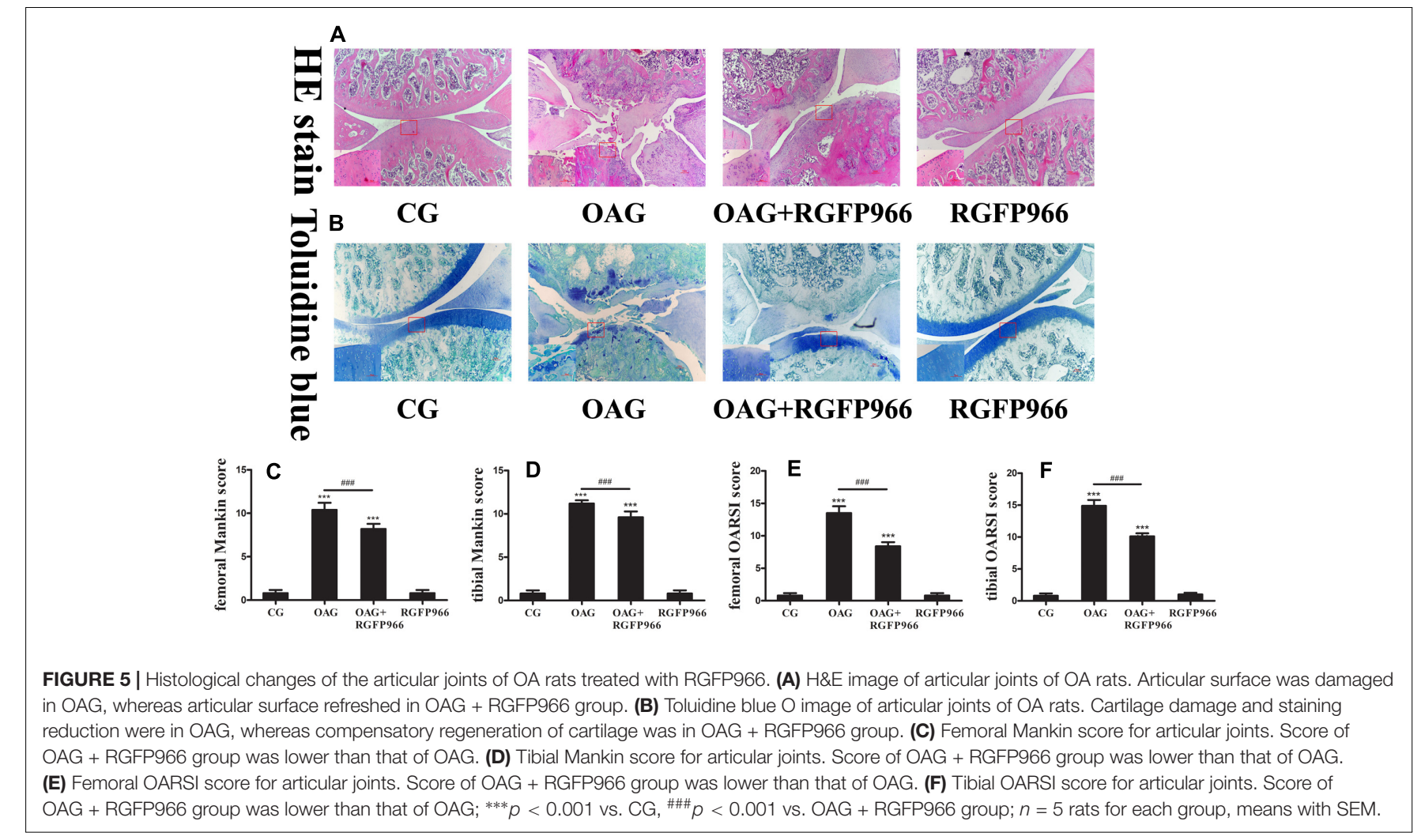

more activity and inflammation (Yang et al., 2018b). We investigated whether RGFP966 could reverse OA progression by regulating NF-kappaB activity. Firstly, we used different concentrations of RGFP966 to stimulate normal chondrocytes. We found that NF-kappaB expression in the cytoplasm of the $10 \mu \mathrm{M}$ group was similar to the control group, and higher than the 1 and $5 \mu \mathrm{M}$ groups, whereas NF-kappaB expression in the nucleus of the $10 \mu \mathrm{M}$ group was lower compared to the other groups (Figures 8A-C,E). HDAC3 levels in the cytoplasm and nucleus of the $10 \mu \mathrm{M}$ group also decreased significantly, compared with the other groups, while there was no significant difference between the $10 \mu \mathrm{M}$ and control groups (Figures $\mathbf{8 A}, \mathbf{B}, \mathbf{D}, \mathbf{F}$ ). We then identified the effect of RGFP966 on IL-1 $\beta$-stimulated chondrocytes. We demonstrated that MMP-13 expression increased in the IL- $1 \beta$ group, and decreased significantly after RGFP966 stimulation (Figures 8G,H). We found that IL- $1 \beta$ could elevate NF-kappaB expression in both the cytoplasm and nucleus of chondrocytes, whereas RGFP966 could reverse this effect (Figures 8G,I,K). We also found increased levels of HDAC3 in both the cytoplasm and nucleus upon IL- $1 \beta$ stimulation, and the increased HDAC3 expression in the nucleus could be reversed by RGFP966 (Figures 8G,J,L). We therefore conclude that RGFP966 could reduce HDAC3 expression in the nucleus in chondrocytes and inhibit the process of NF-kappaB relocation into the nucleus. Furthermore, we determined that HDAC3 could interact with NF-kappaB using co-IP, which suggested HDAC3 may regulate translocation of NF-kappaB by interaction with each other (Supplementary Figure S2).

\section{RGFP966 Could Inhibit ROS Production in IL-1 $\beta$-Stimulated Chondrocytes}

Reactive oxygen species is a pro-inflammatory factor in many signaling pathways, and NF-kappaB is the downstream target of ROS (Yang et al., 2018a). It has been reported that ROS can also cause OA (Lepetsos and Papavassiliou, 2016). Upon IL-1 $\beta$ stimulation, the level of ROS in chondrocytes sharply increased, which was able to activate a series of inflammatory processes including the NF-kappaB signaling pathway. Conversely, RGFP966 could attenuate inflammation by inhibiting ROS production (Figure 9).

\section{DISCUSSION}

The etiology of OA is currently unclear, and consists of many factors such as genetics, environment, and habits (Martelpelletier et al., 2016). It is therefore important to determine the pathogenesis of OA. In our study, we found that HDAC3 is involved in the rat OA model in a time-dependent manner. In addition, moderate-intensity treadmill exercise could protect cartilage and inhibit the inflammatory response in OA. The protective effect of moderateintensity treadmill exercise could be achieved by inhibiting HDAC3/NF-kappaB pathway. To further investigate the underlying mechanism of HDAC3 in OA, we successfully isolated primary rat chondrocytes followed by stimulation with IL-1 $\beta$ to mimic OA-like chondrocytes. We found 


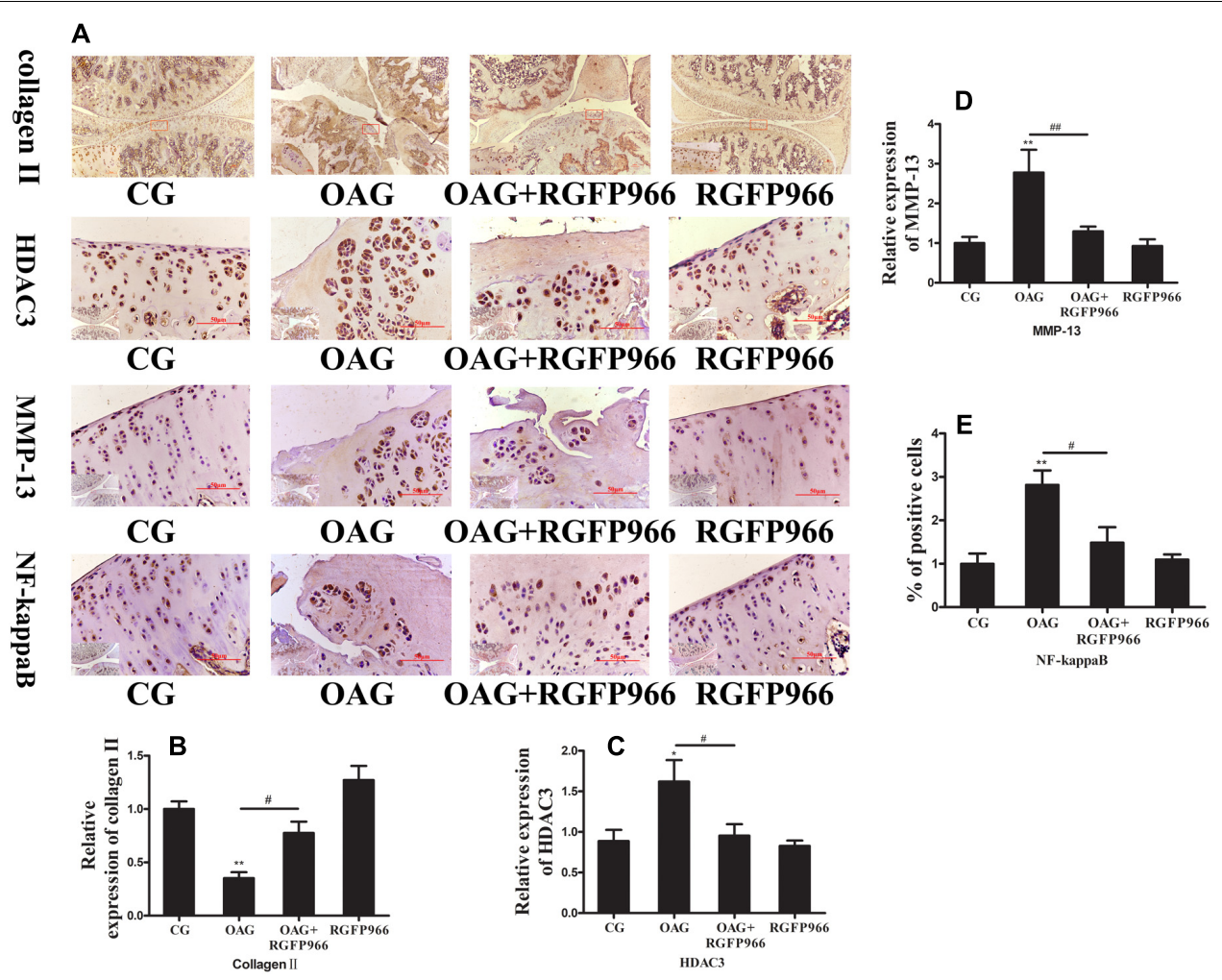

FIGURE 6 | Osteoarthritis rats were treated with RGFP966. Immunohistochemistry (IHC) was performed to measure relative inflammatory proteins in OA. (A,B) Expression of collagen II in CG and OAG + RGFP966 group was higher than OAG by IHC. (A,C) Expression of HDAC3 in CG and OAG + RGFP966 group was lower than OAG by IHC. (A,D) Expression of MMP-13 in CG and OAG + RGFP966 group was lower than OAG by IHC. (A,E) The percentage of NF-kappaB positive cell in $\mathrm{CG}$ and OAG + RGFP966 group were lower than OAG by IHC; ** $p<0.01 ;{ }^{*} p<0.05$ vs. CG, \#\# $p<0.01$; $p<0.05$ vs. OAG + RGFP966 group; $n=5$ rats for each group in $\mathrm{HC}$, means with SEM.

A

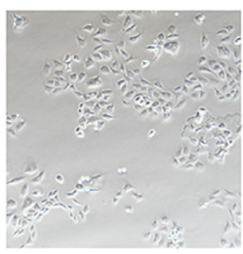

B

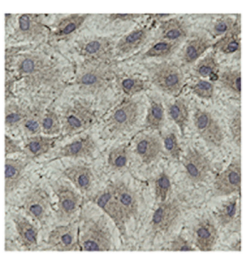

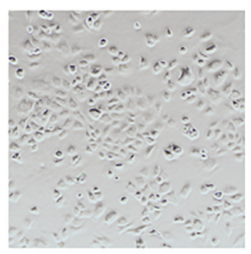
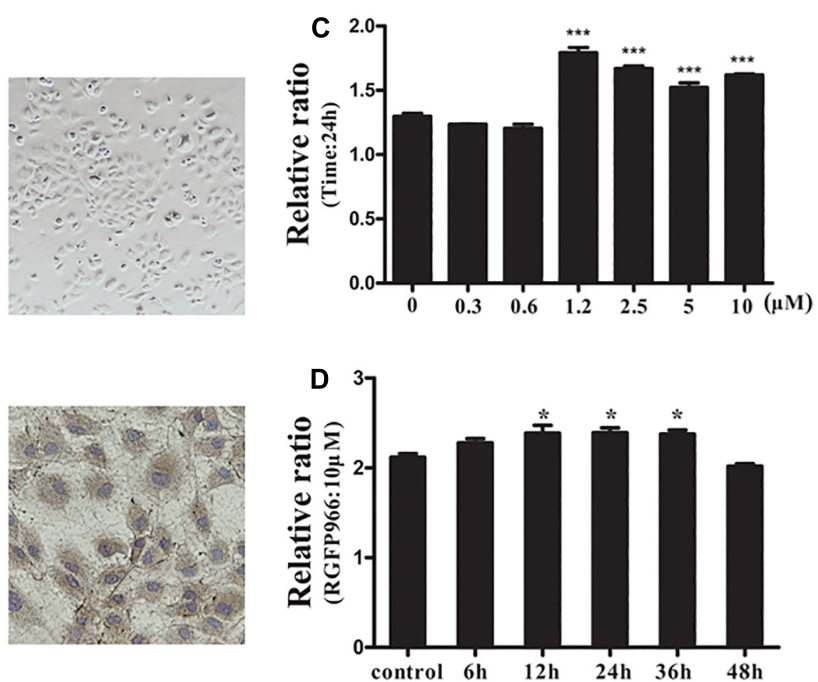

FIGURE 7 | Isolation and identification of primary rat chondrocytes and the effect of the HDAC3 inhibitor RGFP966 on chondrocyte viability. (A) Primary rat chondrocytes were isolated from 4-week old male rats and the chondrocyte morphology was polygon. (B) Primary rat chondrocytes were stained with collagen II antibody for identification, almost all cells were stained with collagen II. (C) The effect of different concentrations of RGFP966 on chondrocyte viability with the same duration, RGFP966 with concentration range between 1.2 and $10 \mu \mathrm{M}$ could promote chondrocyte viability for 24 h. (D) The effect of $10 \mu \mathrm{M}$ RGFP966 on chondrocyte viability in a time-dependent manner, RGFP966 with time range between 12 and 36 h could promote chondrocyte viability with $10 \mu \mathrm{M}$ concentration; ${ }^{* * *} p<0.001 ;{ }^{* *} p<0.01 ;{ }^{*} p<0.05$ vs. control group; $n=3$, means with SEM. 


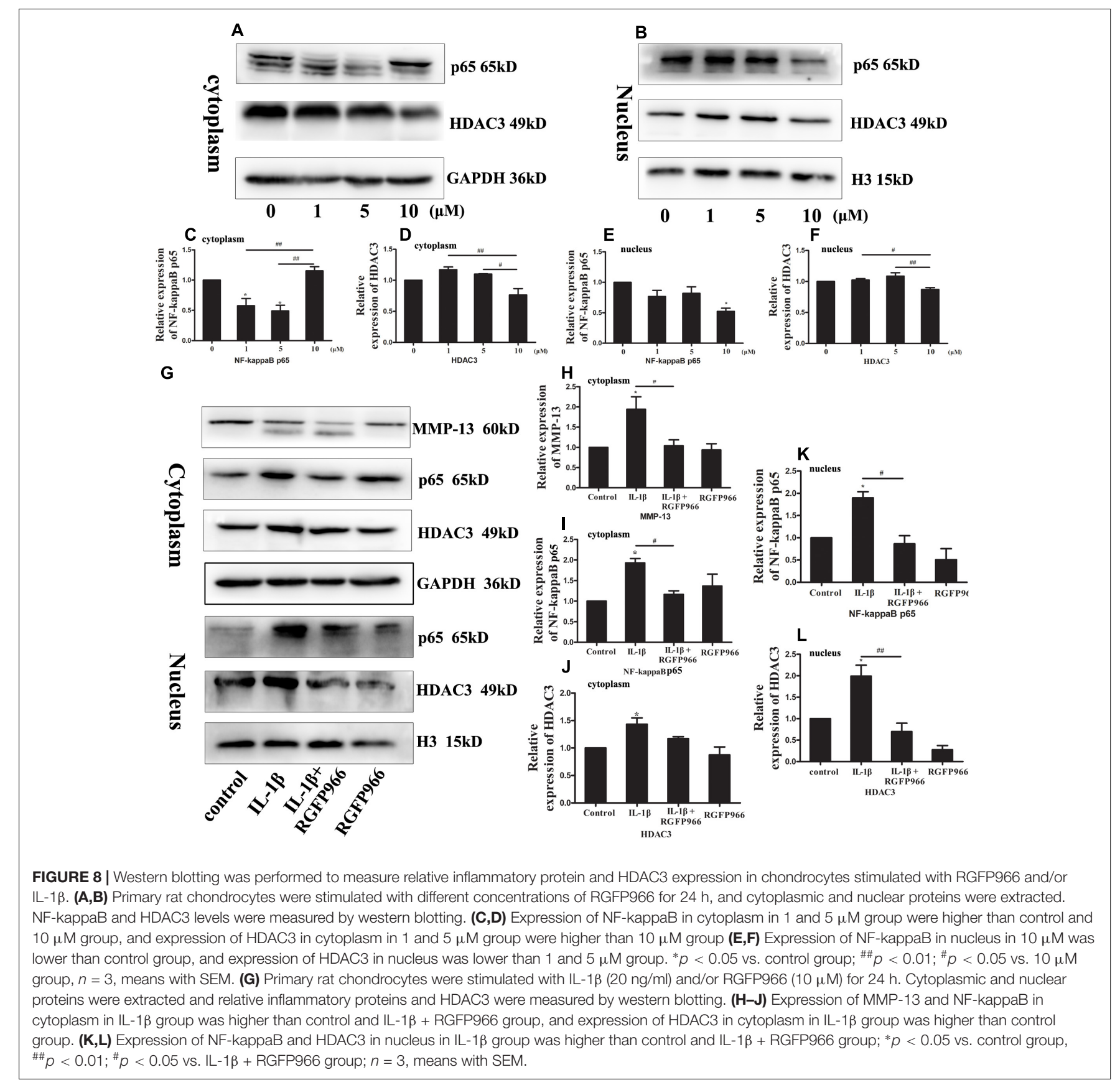

RGFP966, an HDAC3-specific inhibitor, could alleviate IL$1 \beta$-induced inflammation and ROS by inhibition of nuclear translocation of NF-kappaB in chondrocyte. Furthermore, we also revealed RGFP966 could also alleviate inflammation in rat OA model.

Physical exercise has been considered as a new therapy for OA (McAlindon et al., 2014), our results indeed revealed that moderate intensity of treadmill exercise could prevent cartilage from damage, refresh OA cartilage and promote collagen II regeneration, consistent with previous studies ( $\mathrm{Na}$ et al., 2014; Iijima et al., 2015). In addition, NF-kappaB is an important inflammatory transcription factor that promotes MMP-13 and
ADAMTS5 expression resulting in cartilage matrix degradation and cartilage destruction (Roman-Blas and Jimenez, 2006; Wang et al., 2017; Yang et al., 2017). In our study, moderateintensity treadmill exercise could reduce translocation of NFkappaB into the nucleus in the rat OA model. It has been reported that elevated $\mathrm{HDAC} 3$ was found in the cartilage of OA patients (Meng et al., 2018). However, the role of HDAC3 in OA was still unclear. In the manuscript, we found that moderate-intensity treadmill exercise could decrease HDAC3 expression and promote nuclear export of HDAC3 in the rat OA model. Researchers have found that mechanical intervention or physical activity can regulate HDACs expression, also 

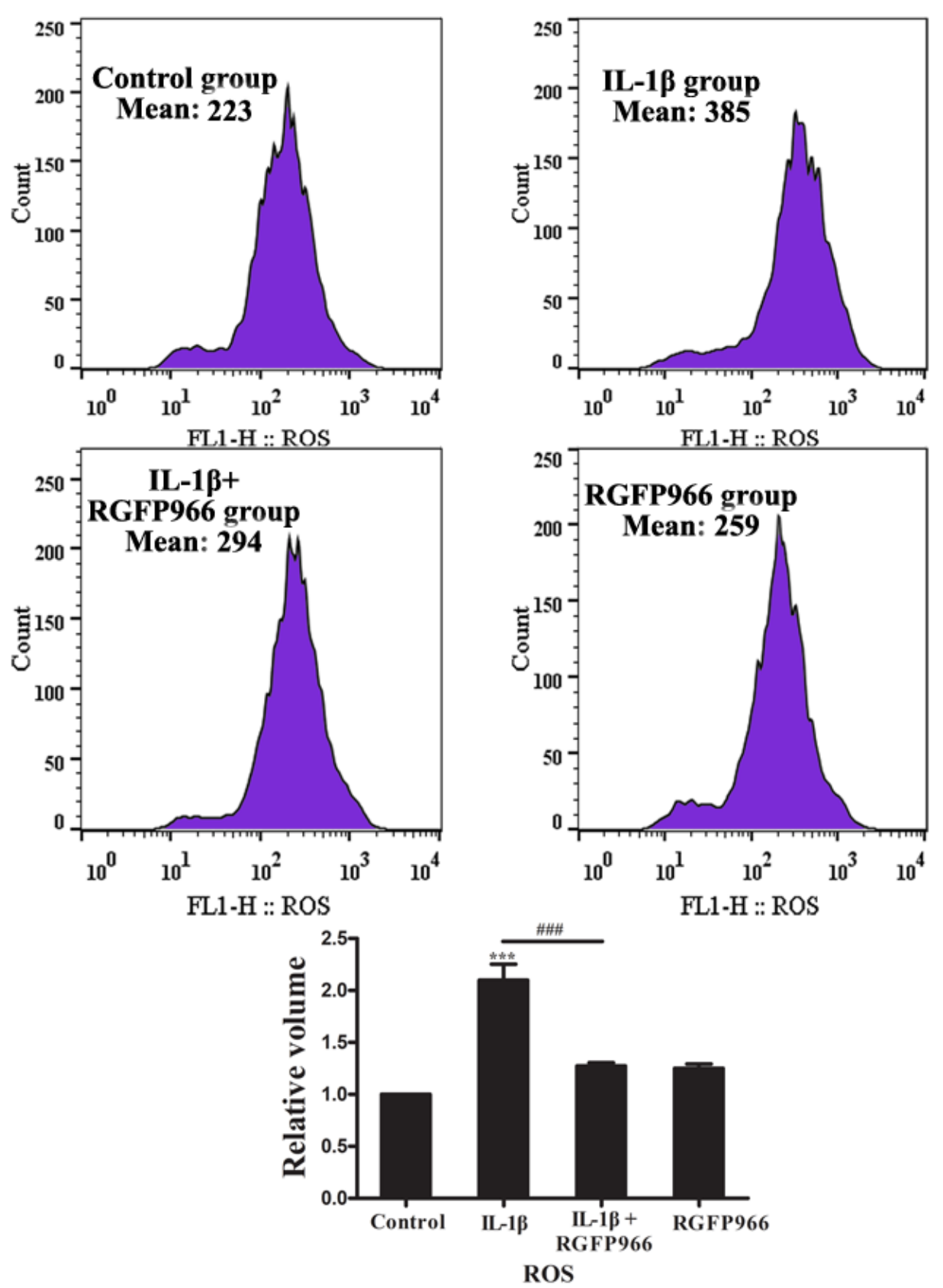

FIGURE 9 | The effect of RGFP966 on ROS production in chondrocytes stimulated with IL-1 $\beta$ (20 ng/ml). Flow cytometry of ROS production in primary rat chondrocytes stimulated with IL-1 $\beta$ (20 ng/ml) and/or RGFP966 (10 $\mu \mathrm{M})$ for $24 \mathrm{~h}$, ROS production in IL-1 $\beta$ group was higher than control and IL-1 $\beta+$ RGFP966 group; ${ }^{* * *} p<0.001,{ }^{*} p<0.05$ vs. control group, ${ }^{\# \# \# ~} p<0.001$ vs. IL-1 $\beta$ + RGFP966 group; $n=3$, means with SEM.

called mechanic-epigenetics. Appropriate exercise could inhibit HDACs expression (Sleiman et al., 2016), promote nuclear export of HDACs (Yuan et al., 2014) and inhibit HDACs activity (Spindler et al., 2014; Fu et al., 2019). However, the mechanism of reducing HDAC3 and the nuclear export of HDAC3 have not been demonstrated. According to previous articles, we suggested a possible mechanism, namely that degradation of HDACs may be caused by increased E3 ubiquitin ligase-induced degradation by the proteasome during exercise
(McGee and Hargreaves, 2011). Exercise may also modulate phosphorylation of HDACs or an association between HDACs with other molecules, such as 14-3-3, which may also promote nuclear export of HDACs (Yuan et al., 2014). However, whether HDAC3 synthesis is also inhibited is unclear. Interestingly, highintensity exercise was not able to protect cartilage in the rat OA model. The following mechanisms could be involved in this phenomenon: activation of osteoblasts may increase the subchondral bone mass to adapt to the increased physical load 
exposure, which could cause cartilage damage (Siebelt et al., 2014); the mechanobiology homeostasis of cartilage depends on the balance of hydrostatic stress and octahedral sheer stress. When overloaded on the knee, this balance is disturbed, resulting in mechanical damage of cartilage (Carter et al., 2004). Moreover, excessive ROS production may also contribute to OA with high-intensity exercise. Low levels of ROS are essential for normal cartilage and appropriate exercise could maintain the balance between oxidative and anti-oxidative system (Finaud et al., 2006). When cartilage is overloaded, the balance is broken and elevated ROS will cause injury to cartilage (Cooper et al., 2002; Baur et al., 2011). This phenomenon is similar to that in vitro, extreme axial compression increased oxidative stress in chondrocytes (Beecher et al., 2007). Meanwhile, the symptoms of OA were also alleviated by RGFP966 in vivo; the expression of MMP-13 and NF-kappaB decreased significantly in OA rats treated with RGFP966. Histological changes and the score system also represented the protective effect of RGFP966 in vivo.

To further investigate the mechanism of HDAC3 in OA in vitro, we isolated primary rat chondrocytes and used RGFP966 at the basis of IL-1 $\beta$ stimulation to explore the effect of HDAC3 in OA. We found that RGFP966 could promote chondrocyte viability, and that high concentration of RGFP966 could significantly decrease the level of HDAC3 in the nucleus in normal chondrocytes, suggesting that the effect of HDAC3 on OA was dose-dependent. We also found elevated MMP-13 level and nuclear expression of NF-kappaB and HDAC3 was increased in chondrocytes stimulated with IL-1 $\beta$, which could be reversed by RGFP966. When chondrocytes were stimulated with TNF- $\alpha$ instead of IL- $1 \beta$ we revealed the similar phenomenon (Supplementary Figures S7, S8).

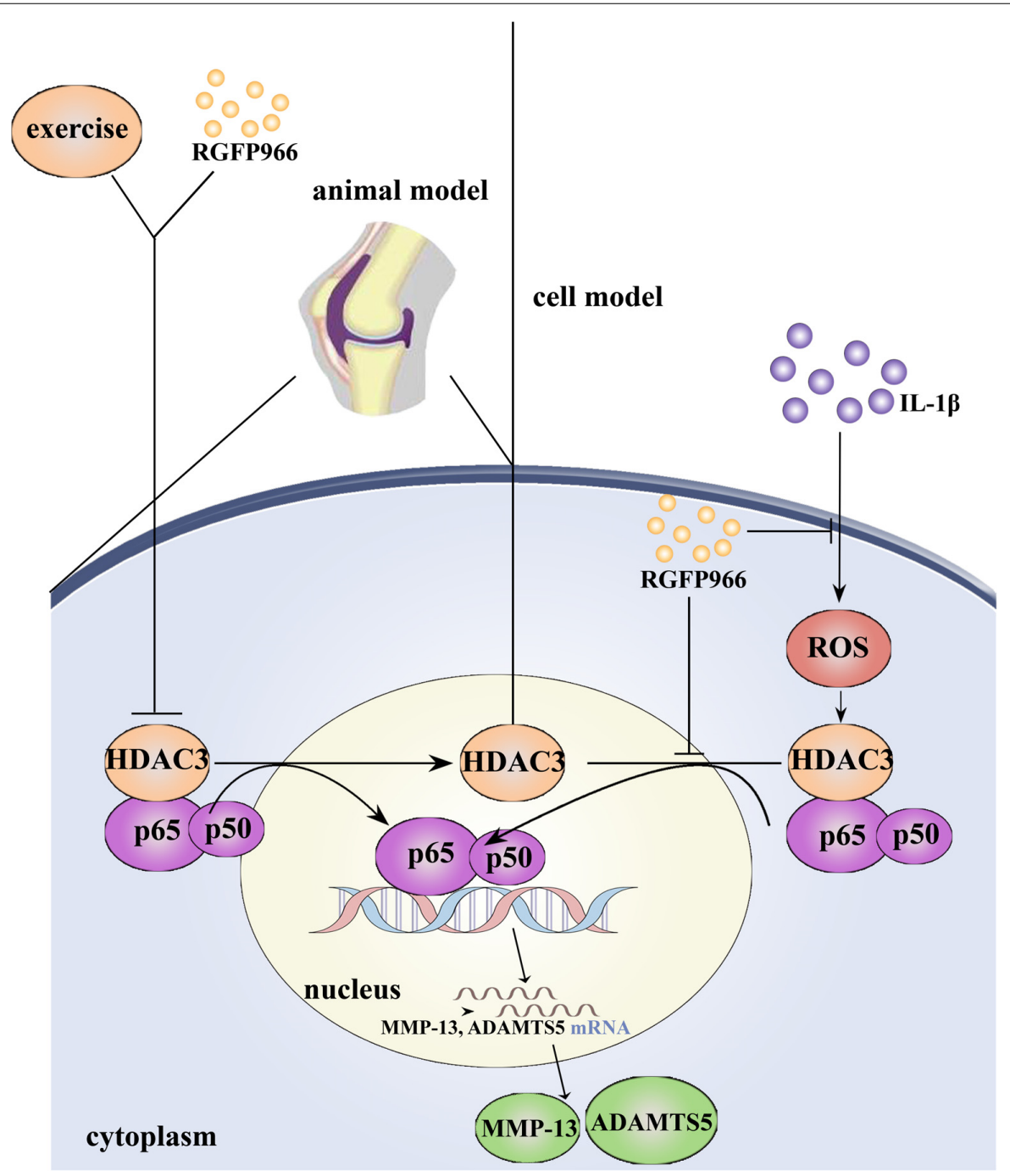

FIGURE 10 | Schematic diagram of the mechanisms about the role of HDAC3/NF-kappaB pathway in pathogenesis and exercise therapy of OA. Moderate-intensity of exercise can inhibit nuclear translocation of HDAC3/NF-kappaB complex, leading to decreased expression of inflammatory proteins. Meanwhile, the HDAC3 inhibitor RGFP966 could also alleviate inflammation response in OA through HDAC3/NF-kappaB pathway in vivo and in vitro. 
Accordingly, we suggest that $\mathrm{HDAC} 3$ is a pro-inflammatory factor in OA. The mechanism of HDAC3 in OA could be that HDAC3 regulates relative inflammatory gene expression (Angiolilli et al., 2017), and NF-kappaB activity (Leus et al., 2016). Interestingly, we found that the change in nuclear NF-kappaB and HDAC3 always occurred simultaneously. We therefore hypothesized that HDAC3 could associate with NF-kappaB translocation into the nucleus as a co-factor and promote NF-kappaB activity. To confirm this potential interaction, we investigated co-immunoprecipitation of HDAC3 with NF-kappaB. This finding was also supported by a database $^{1}$ (Supplementary Figure S3). In previous studies, TNF- $\alpha$ stimulation was shown to enhance HDAC3-NF-kappaB complex translocation into the nucleus in 3T3-L1 adipocytes (Gao et al., 2006), and TNF- $\alpha$ also facilitated HDAC3 and NF-kappaB protein expression and nuclear translocation in A549 cells simultaneously in lung inflammation (Pooladanda et al., 2019). Furthermore, unphosphorylated NF-kappaB but not NF-kappaB phosphorylated at S536, was shown to bind to HDAC3 (Hu et al., 2004). A possible mechanism is that HDAC3 binds to unphosphorylated NF-kappaB in the cytoplasm and is translocated to the nucleus upon inflammatory stimulation (Pooladanda et al., 2019). Meanwhile, NF-kappaB separates from HDAC3 followed by S536 phosphorylation occurs. Another possible mechanism is that HDAC3 could change the acetylation level of NF-kappaB in the nucleus. $\mathrm{CBP} / \mathrm{p} 300$ could promote NF-kappaB nuclear export through elevated levels of acetylation, whereas HDAC3 reverses this process (Kiernan et al., 2003). Meanwhile, RGFP966 might inhibit HDAC3/NFkappaB pathway though above mentioned mechanism. However, the underlying mechanism of how HDAC3 can regulate NFkappaB activity requires further investigation. ROS is a proinflammatory factor that can activate NF-kappaB in OA. We found that HDAC3 may also promote ROS production and activate NF-kappaB signaling via the ROS/NF-kappaB axis. It was reported HDAC3 promoted ROS production by regulating acetylation of Nrf2/ARE signaling (Chen X. et al., 2018). It is thus well-accepted that RGFP966 could inhibit ROS production in chondrocytes under IL-1 $\beta$ stimulation, which was demonstrated in a previous article (Zhao et al., 2019). We have therefore constructed a schematic diagram to summarize our experiments (Figure 10).

There are some limitations of our study. Firstly, it is not clear whether RGFP966 can substitute moderate exercise and there are possible side effects when administered to OA patients. Secondly, in this study, we only focused on changes in cartilage and chondrocyte. We should determine the mechanisms of cross-talk

${ }^{1}$ https://string-db.org

\section{REFERENCES}

Angiolilli, C., Kabala, P. A., Grabiec, A. M., Van Baarsen, I. M., Ferguson, B. S., Garcia, S., et al. (2017). Histone deacetylase 3 regulates the inflammatory gene expression programme of rheumatoid arthritis fibroblast-like synoviocytes. Ann. Rheum. Dis. 76, 277-285. doi: 10.1136/annrheumdis-2015-209064 and feedback among other articular tissues (Loeser et al., 2012). Finally, it should be mentioned that we defined the different intensities of exercise according to the different percentages of $\mathrm{VO}_{2 \max }$ for SD rats (Bedford et al., 1979), but these results have not been translated into a clinical setting due to individual differences.

\section{DATA AVAILABILITY}

The raw data supporting the conclusions of this manuscript will be made available by the authors, without undue reservation, to any qualified researcher.

\section{ETHICS STATEMENT}

This study was carried out in accordance with the principles of recommendations of U.K. Animals (Scientific Procedures) Act, Ethics Committee of China Medical University. The protocol was approved by the Ethics Committee of China Medical University.

\section{AUTHOR CONTRIBUTIONS}

XZ conceived the study. YY developed the methodology. YW developed the software. LJ performed the formal analysis. YG investigated the results. JL provided the resources. $\mathrm{HZ}$ curated the data and drafted the manuscript. LB reviewed and edited the manuscript. All authors approved the final manuscript.

\section{FUNDING}

This research was funded by the National Natural Science Foundation of China, grant numbers 81772420 and 81272050 .

\section{ACKNOWLEDGMENTS}

We appreciate the help from our fellows in the lab affiliated to China Medical University, Liaoning, China.

\section{SUPPLEMENTARY MATERIAL}

The Supplementary Material for this article can be found online at: https://www.frontiersin.org/articles/10.3389/fphys. 2019.01060/full\#supplementary-material

Ashkavand, Z., Malekinejad, H., and Vishwanath, B. S. (2013). The pathophysiology of osteoarthritis. J. Phar. Res. 7, 132-138.

Baur, A., Henkel, J., Bloch, W., Treiber, N., Scharffetter-Kochanek, K., Bruggemann, G. P., et al. (2011). Effect of exercise on bone and articular cartilage in heterozygous manganese superoxide dismutase (SOD2) deficient mice. Free Radic. Res. 45, 550-558. doi: 10.3109/10715762.2011.555483 
Bedford, T. G., Tipton, C. M., Wilson, N. C., Oppliger, R. A., and Gisolfi, C. V. (1979). Maximum oxygen consumption of rats and its changes with various experimental procedures. J. Appl. Physiol. Respir. Environ. Exerc. Physiol. 47, 1278-1283. doi: 10.1152/jappl.1979.47.6.1278

Beecher, B. R., Martin, J. A., Pedersen, D. R., Heiner, A. D., and Buckwalter, J. A. (2007). Antioxidants block cyclic loading induced chondrocyte death. Iowa. Orthop. J. 27, 1-8.

Berenbaum, F. (2013). Osteoarthritis as an inflammatory disease (osteoarthritis is not osteoarthrosis!). Osteoarthr. Cartil. 21, 16-21. doi: 10.1016/j.joca.2012. 11.012

Boudenot, A., Presle, N., Uzbekov, R., Toumi, H., Pallu, S., and Lespessailles, E. (2014). Effect of interval-training exercise on subchondral bone in a chemicallyinduced osteoarthritis model. Osteoarthr. Cartil. 22, 1176-1185. doi: 10.1016/j. joca.2014.05.020

Carpio, L. R., Bradley, E. W., Mcgeelawrence, M. E., Weivoda, M. M., Poston, D. D., Dudakovic, A., et al. (2016). Histone deacetylase 3 supports endochondral bone formation by controlling cytokine signaling and matrix remodeling. Sci. Signal. 9:ra79. doi: 10.1126/scisignal.aaf3273

Carpio, L. R., and Westendorf, J. J. (2016). Histone deacetylases in cartilage homeostasis and osteoarthritis. Curr. Rheumatol. Rep. 18:52. doi: 10.1007/ s11926-016-0602-z

Carter, D. R., Beaupr, G. S., Wong, M., Smith, R. L., Andriacchi, T. P., and Schurman, D. J. (2004). The mechanobiology of articular cartilage development and degeneration. Clin. Orthop. Relat. Res. 427, S69-S77. doi: 10.1097/01.blo. 0000144970.05107.7e

Chang, S. F., Hsieh, R. Z., Huang, K. C., Chang, C. A., Chiu, F. Y., Kuo, H. C., et al. (2015). Upregulation of bone morphogenetic protein2 synthesis and consequent collagen II expression in leptin-stimulated human chondrocytes. PLoS One 10:e0144252. doi: 10.1371/journal.pone. 0144252

Chen, L. F., and Greene, W. C. (2004). Shaping the nuclear action of NF-kappaB. Nat. Rev. Mol. Cell. Biol. 5, 392-401. doi: 10.1038/nrm1368

Chen, S., Ye, J., Chen, X., Shi, J., Wu, W., Lin, W., et al. (2018). Valproic acid attenuates traumatic spinal cord injury-induced inflammation via STAT1 and NF-кB pathway dependent of HDAC3. J. Neuroinflamm. 15:150.

Chen, X., Wang, H., Zhou, M., Li, X., Fang, Z., Gao, H., et al. (2018). Valproic acid attenuates traumatic brain injury-induced inflammation in vivo: involvement of autophagy and the Nrf2/ARE signaling pathway. Front. Mol. Neurosci. 11:117. doi: 10.3389/fnmol.2018.00117

Chen, X., Barozzi, I., Termanini, A., Prosperini, E., Recchiuti, A., Dalli, J., et al. (2012). Requirement for the histone deacetylase Hdac3 for the inflammatory gene expression program in macrophages. Proc. Nat. Acad. Sci. U.S.A. 109, 16768-16769. doi: 10.1073/pnas.1121131109

Cheng, Y. J., Hootman, J. M., Murphy, L. B., Langmaid, G. A., and Helmich, C. G. (2010). Prevalence of doctor-diagnosed arthritis and arthritis-attributable activity limitation - United States, 2007-2009. Mmwr Morb. Mortal. Wkly Rep. 59:1261.

Cifuentes, D. J., Rocha, L. G., Silva, L. A., Brito, A. C., Rueff-Barroso, C. R., Porto, L. C., et al. (2010). Decrease in oxidative stress and histological changes induced by physical exercise calibrated in rats with osteoarthritis induced by monosodium iodoacetate. Osteoarthr. Cartilage 18, 1088-1095. doi: 10.1016/j. joca.2010.04.004

Cooper, C. E., Vollaard, N. B., Choueiri, T., and Wilson, M. T. (2002). Exercise, free radicals and oxidative stress. Biochem. Soc. Trans. 30, 280-285.

Correa, R. O., Vieira, A., Sernaglia, E. M., Lancellotti, M., Vieira, A. T., AvilaCampos, M. J., et al. (2017). Bacterial short-chain fatty acid metabolites modulate the inflammatory response against infectious bacteria. Cell Microbiol. 19. doi: $10.1111 / \mathrm{cmi} .12720$ [Epub ahead of print].

Custers, R. J., Creemers, L. B., Verbout, A. J., van Rijen, M. H., Dhert, W. J., and Saris, D. B. (2007). Reliability, reproducibility and variability of the traditional histologic/histochemical grading system vs the new OARSI osteoarthritis cartilage histopathology assessment system. Osteoarthr. Cartilage 15, 12411248. doi: 10.1016/j.joca.2007.04.017

De Ruijter, A. J. M., Van Gennip, A. H., Caron, H. N., Kemp, S., and Van Kuilenburg, A. B. P. (2003). Histone deacetylases (HDACs): characterization of the classical HDAC family. Biochem. J. 370, 737-749. doi: 10.1042/bj200 21321
Ernest, T. L., and Kondrashov, P. E. (2018). The role of excessive body weight and meniscal instability in the progression of osteoarthritis in a rat model. Knee 25, 1151-1156. doi: 10.1016/j.knee.2018.07.009

Finaud, J., Lac, G., and Filaire, E. (2006). Oxidative stress: relationship with exercise and training. Sports Med. 36, 327-358. doi: 10.2165/00007256-20063604000004

Franciozi, C. E., Tarini, V. A., Reginato, R. D., Gonçalves, P. R., Medeiros, V. P., Ferretti, M., et al. (2013). Gradual strenuous running regimen predisposes to osteoarthritis due to cartilage cell death and altered levels of glycosaminoglycans. Osteoarthr. Cartilage 21, 965-972. doi: 10.1016/j.joca. 2013.04.007

Fu, S., Thompson, C. L., Ali, A., Wang, W., Chapple, J. P., Mitchison, H. M., et al. (2019). Mechanical loading inhibits cartilage inflammatory signalling via an HDAC6 and IFT-dependent mechanism regulating primary cilia elongation. Osteoarthr. Cartilage. 27, 1064-1074. doi: 10.1016/j.joca.2019. 03.003

Gao, Z., He, Q., Peng, B., Chiao, P. J., and Ye, J. (2006). Regulation of nuclear translocation of HDAC3 by IkappaBalpha is required for tumor necrosis factor inhibition of peroxisome proliferator-activated receptor gamma function. J. Biol. Chem. 281, 4540-4547. doi: 10.1074/jbc.M507784200

Gerwin, N., Bendele, A. M., Glasson, S., and Carlson, C. S. (2010). The OARSI histopathology initiative - recommendations for histological assessments of osteoarthritis in the rat. Osteoarthr. Cartilage 18(Suppl. 3), S24-S34. doi: 10. 1016/j.joca.2010.05.030

Hu, J., Nakano, H., Sakurai, H., and Colburn, N. H. (2004). Insufficient p65 phosphorylation at S536 specifically contributes to the lack of NF-kappaB activation and transformation in resistant JB6 cells. Carcinogenesis 25, 19912003. doi: $10.1093 /$ carcin/bgh 198

Huang, X., Xu, J., Huang, M., Li, J., Dai, L., Dai, K., et al. (2014). Histone deacetylasel promotes TGF-beta1-mediated early chondrogenesis through down-regulating canonical Wnt signaling. Biochem. Biophys. Res. Commun. 453, 810-816. doi: 10.1016/j.bbrc.2014.10.021

Iijima, H., Aoyama, T., Ito, A., Yamaguchi, S., Nagai, M., Tajino, J., et al. (2015). Effects of short-term gentle treadmill walking on subchondral bone in a rat model of instability-induced osteoarthritis. Osteoarthr. Cartilage 23, 15631574. doi: 10.1016/j.joca.2015.04.015

Jeong, J. Y., Cha, H. J., Choi, E. O., Kim, C. H., Kim, G. Y., Yoo, Y. H., et al. (2019). Activation of the $\mathrm{Nrf} 2 / \mathrm{HO}-1$ signaling pathway contributes to the protective effects of baicalein against oxidative stress-induced DNA damage and apoptosis in HEI193 Schwann cells. Int. J. Med. Sci. 16, 145-155. doi: 10.7150/ijms.27005

Jia, H., Wang, Y., Morris, C. D., Jacques, V., Gottesfeld, J. M., Rusche, J. R., et al. (2016). The effects of pharmacological inhibition of histone deacetylase 3 (HDAC3) in huntington's disease mice. PLoS One 11:e0152498. doi: 10.1371/ journal.pone. 0152498

Kiernan, R., Bres, V., Ng, R. W., Coudart, M. P., El Messaoudi, S., Sardet, C., et al. (2003). Post-activation turn-off of NF-kappa B-dependent transcription is regulated by acetylation of p65. J. Biol. Chem. 278, 2758-2766. doi: 10.1074/ jbc.M209572200

Lee, Y. J., Park, J. A., Yang, S. H., Kim, K. Y., Kim, B. K., Lee, E. Y., et al. (2011). Evaluation of osteoarthritis induced by treadmill-running exercise using the modified Mankin and the new OARSI assessment system. Rheumatol. Int. 31, 1571-1576. doi: 10.1007/s00296-010-1520-4

Lepetsos, P., and Papavassiliou, A. G. (2016). ROS/oxidative stress signaling in osteoarthritis. Biochim. Biophys. Acta. 1862, 576-591. doi: 10.1016/j.bbadis. 2016.01.003

Leus, N. G., van der Wouden, P. E., van den Bosch, T., Hooghiemstra, W. T., Ourailidou, M. E., Kistemaker, L. E., et al. (2016). HDAC 3-selective inhibitor RGFP966 demonstrates anti-inflammatory properties in RAW 264.7 macrophages and mouse precision-cut lung slices by attenuating NF-kappaB p65 transcriptional activity. Biochem. Pharmacol. 108, 58-74. doi: 10.1016/j.bcp. 2016.03.010

Loeser, R. F., Goldring, S. R., Scanzello, C. R., and Goldring, M. B. (2012). Osteoarthritis: a disease of the joint as an organ. Arthritis Rheum 64, 1697-1707. doi: 10.1002/art.34453

Malvaez, M., McQuown, S. C., Rogge, G. A., Astarabadi, M., Jacques, V., Carreiro, S., et al. (2013). HDAC3-selective inhibitor enhances extinction of cocaineseeking behavior in a persistent manner. Proc. Natl. Acad. Sci. U.S.A. 110, 2647-2652. doi: 10.1073/pnas.1213364110 
Martelpelletier, J., Barr, A. J., Cicuttini, F. M., Conaghan, P. G., Cooper, C., Goldring, M. B., et al. (2016). Osteoarthritis. Nat. Rev. Dis. Primers 85:84.

McAlindon, T. E., Bannuru, R. R., Sullivan, M. C., Arden, N. K., Berenbaum, F., Bierma-Zeinstra, S. M., et al. (2014). OARSI guidelines for the non-surgical management of knee osteoarthritis. Osteoarthr. Cartilage 22, 363-388. doi: 10.1016/j.joca.2014.01.003

McGee, S. L., and Hargreaves, M. (2011). Histone modifications and exercise adaptations. J. Appl. Physiol. 110, 258-263. doi: 10.1152/japplphysiol.00979. 2010

Meng, F., Li, Z., Zhang, Z., Yang, Z., Kang, Y., Zhao, X., et al. (2018). MicroRNA-193b-3p regulates chondrogenesis and chondrocyte metabolism by targeting HDAC3. Theranostics 8, 2862-2883. doi: 10.7150/thno. 23547

Mobasheri, A., and Batt, M. (2016). An update on the pathophysiology of osteoarthritis. Ann. Phys. Rehabil. Med. 59, 333-339. doi: 10.1016/j.rehab.2016. 07.004

Na, S. S., Kim, S. G., Yong, M. S., and Hwangbo, G. (2014). Study of treadmill exercise effect on rats with osteoarthritis using proteomic analysis. J. Phys. Ther. Sci. 26, 487-490. doi: 10.1589/jpts.26.487

Ni, G. X., Liu, S. Y., Lei, L., Li, Z., Zhou, Y. Z., and Zhan, L. Q. (2013). Intensitydependent effect of treadmill running on knee articular cartilage in a rat model. BioMed. Res. Int. 2013:172392. doi: 10.1155/2013/172392

Pooladanda, V., Thatikonda, S., Bale, S., Pattnaik, B., Sigalapalli, D. K., Bathini, N. B., et al. (2019). Nimbolide protects against endotoxin-induced acute respiratory distress syndrome by inhibiting TNF-alpha mediated NF-kappaB and HDAC-3 nuclear translocation. Cell Death Dis. 10:81. doi: 10.1038/s41419018-1247-9

Roman-Blas, J. A., and Jimenez, S. A. (2006). NF-кB as a potential therapeutic target in osteoarthritis and rheumatoid arthritis. Osteoarthr. Cartilage 14, 839-848. doi: 10.1016/j.joca.2006.04.008

Saito, R., Muneta, T., Ozeki, N., Nakagawa, Y., Udo, M., Yanagisawa, K., et al. (2017). Strenuous running exacerbates knee cartilage erosion induced by low amount of mono-iodoacetate in rats. BMC Musculoskelet. Disord. 18:36. doi: 10.1186/s12891-017-1393-8

Schmitt, H. M., Schlamp, C. L., and Nickells, R. W. (2018). Targeting HDAC3 activity with RGFP966 protects against retinal ganglion cell nuclear atrophy and apoptosis after optic nerve injury. J. Ocul. Pharmacol. Ther. 34, 260-273. doi: 10.1089/jop.2017.0059

Sekiya, I., Tang, T., Hayashi, M., Morito, T., Ju, Y. J., Mochizuki, T., et al. (2009). Periodic knee injections of BMP-7 delay cartilage degeneration induced by excessive running in rats. J. Orthop. Res. 27, 1088-1092. doi: 10.1002/jor. 20840

Siebelt, M., Groen, H. C., Koelewijn, S. J., de Blois, E., Sandker, M., Waarsing, J. H., et al. (2014). Increased physical activity severely induces osteoarthritic changes in knee joints with papain induced sulfate-glycosaminoglycan depleted cartilage. Arthritis Res. Ther. 16:R32. doi: 10.1186/ar4461

Sleiman, S. F., Henry, J., Al-Haddad, R., El Hayek, L., Abou Haidar, E., Stringer, T., et al. (2016). Exercise promotes the expression of brain derived neurotrophic factor (BDNF) through the action of the ketone body beta-hydroxybutyrate. Elife 5:e15092. doi: 10.7554/eLife.15092

Song, J., Jin, E. H., Dongkyun, K., Young, K. K., Churl-Hong, C., and Jin, E. J. (2015). MicroRNA-222 regulates MMP-13 via targeting HDAC-4 during osteoarthritis pathogenesis. BBA Clin. 3, 79-89. doi: 10.1016/j.bbacli.2014. 11.009

Spindler, C., Cechinel, L. R., Basso, C., Moyses, F., Bertoldi, K., Roesler, R., et al. (2014). Treadmill exercise alters histone acetyltransferases and histone deacetylases activities in frontal cortices from wistar rats. Cell Mol. Neurobiol. 34, 1097-1101. doi: 10.1007/s10571-014-0096-z

Su, Y. P., Chen, C. N., Huang, K. C., Chang, H. I., Lee, K. C., Lo, C. M., et al. (2018). Leptin induces MMP1/13 and ADAMTS 4 expressions through bone morphogenetic protein-2 autocrine effect in human chondrocytes. J. Cell Biochem. 119, 3716-3724. doi: 10.1002/jcb.26593

Udo, M., Muneta, T., Tsuji, K., Ozeki, N., Nakagawa, Y., Ohara, T., et al. (2016). Monoiodoacetic acid induces arthritis and synovitis in rats in a dose- and time-dependent manner: proposed model-specific scoring systems. Osteoarthr. Cartilage 24, 1284-1291. doi: 10.1016/j.joca.2016.02.005

Wang, Z., Huang, J., Zhou, S., Luo, F., Xu, W., Wang, Q., et al. (2017). Anemonin attenuates osteoarthritis progression through inhibiting the activation of IL1ß/NF-кB pathway. J. Cell. Mol. Med. 21, 3231-3243. doi: 10.1111/jcmm. 13227

Wei, Y., Wang, Y., Wang, Y., and Bai, L. (2017). Transient receptor potential vanilloid 5 mediates $\mathrm{Ca} 2+$ influx and inhibits chondrocyte autophagy in a rat osteoarthritis model. Cell. Physiol. Biochem. 42, 319-332. doi: 10.1159/ 000477387

Yang, Y., Wang, Y., Kong, Y., Zhang, X., and Bai, L. (2017). The effects of different frequency treadmill exercise on lipoxin A4 and articular cartilage degeneration in an experimental model of monosodium iodoacetate-induced osteoarthritis in rats. Plos One 12:e0179162. doi: 10.1371/journal.pone.0179162

Yang, Y., Wang, Y., Kong, Y., Zhang, X., Zhang, H., Gang, Y., et al. (2018a). Carnosine prevents type 2 diabetes-induced osteoarthritis through the ROS/NF-kappaB pathway. Front. Pharmacol. 9:598. doi: 10.3389/fphar.2018. 00598

Yang, Y., Wang, Y., Kong, Y., Zhang, X., Zhang, H., Gang, Y., et al. (2018b). Mechanical stress protects against osteoarthritis via regulation of the AMPK/NF-kappaB signaling pathway. J. Cell. Physiol. 234, 9156-9167. doi: $10.1002 /$ jcp. 27592

Yang, Y., Wang, Y., Kong, Y., Zhang, X., Zhang, H., Gang, Y., et al. (2018c). The therapeutic effects of lipoxin A4 during treadmill exercise on monosodium iodoacetate-induced osteoarthritis in rats. Mol. Immunol. 103, 35-45. doi: 10. 1016/j.molimm.2018.08.027

Yuan, H., Niu, Y., Liu, X., and Fu, L. (2014). Exercise increases the binding of MEF2A to the Cpt1b promoter in mouse skeletal muscle. Acta. Physiol. 212, 283-292. doi: 10.1111/apha.12395

Zhang, X., Yang, Y., Li, X., Zhang, H., Gang, Y., and Bai, L. (2019). Alterations of autophagy in knee cartilage by treatment with treadmill exercise in a rat osteoarthritis model. Int. J. Mol. Med. 43, 336-344. doi: 10.3892/ijmm.2018. 3948

Zhao, B., Yuan, Q., Hou, J. B., Xia, Z. Y., Zhan, L. Y., Li, M., et al. (2019). Inhibition of HDAC3 ameliorates cerebral ischemia reperfusion injury in diabetic mice in vivo and in vitro. J. Diabetes Res. 2019:8520856. doi: 10.1155/2019/8520856

Zhu, H., Shan, L., Schiller, P. W., Mai, A., and Peng, T. (2010). Histone deacetylase3 activation promotes tumor necrosis factor-alpha (TNF-alpha) expression in cardiomyocytes during lipopolysaccharide stimulation. J. Biol. Chem. 285, 9429-9436. doi: 10.1074/jbc.M109.071274

Ziesche, E., Kettner-Buhrow, D., Weber, A., Wittwer, T., Jurida, L., Soelch, J., et al. (2013). The coactivator role of histone deacetylase 3 in IL-1-signaling involves deacetylation of p65 NF-kappaB. Nucleic Acids Res. 41, 90-109. doi: $10.1093 /$ nar/gks916

Conflict of Interest Statement: The authors declare that the research was conducted in the absence of any commercial or financial relationships that could be construed as a potential conflict of interest.

Copyright (C) 2019 Zhang, Ji, Yang, Wei, Zhang, Gang, Lu and Bai. This is an openaccess article distributed under the terms of the Creative Commons Attribution License (CC BY). The use, distribution or reproduction in other forums is permitted, provided the original author(s) and the copyright owner(s) are credited and that the original publication in this journal is cited, in accordance with accepted academic practice. No use, distribution or reproduction is permitted which does not comply with these terms. 